\title{
Term definable classes of Boolean functions and frame definability in modal logic
}

\author{
M. Couceiro, L. Hella, J. Kivelä
}

\begin{abstract}
We establish a connection between term definability of Boolean functions and definability of finite modal frames. We introduce a bijective translation between functional terms and uniform degree- 1 formulas and show that a class of Boolean functions is defined by functional terms if and only if the corresponding class of Scott-Montague frames is defined by the translations of these functional terms, and vice versa. As a special case, we get that the clone $\Lambda_{1}$ of all conjunctions corresponds to the class of all Kripke frames. We also characterize some classes of Scott-Montague frames corresponding to subclones of $\Lambda_{1}$ by restricting the class of Kripke frames in a natural way. Furthermore, by modifying Kripke semantics, we extend our results to correspondences between linear clones and classes of Kripke frames equipped with modified semantics.
\end{abstract}

\section{Introduction}

In [16] Segerberg studied a semantics for modal logic which is more general than the well-known Kripke semantics. In literature this semantics is often called neighbourhood semantics. Hansson and Gärdenfors formulated the same semantics in a different, yet an equivalent, way in [9]. Following them, we call this semantics Scott-Montague semantics and here we also adopt their definition for the semantics.

A Scott-Montague frame is a structure $\langle W, F\rangle$ where $W$ is a non-empty set and $F$ is a set function $\mathcal{P}(W) \rightarrow \mathcal{P}(W)$. The truth condition for the modal operator $\square$ in a model $\mathcal{M}$ based on a Scott-Montague frame is given by

$$
\mathcal{M}, w=\square \varphi \text { if and only if } w \in F\left(\|\varphi\|^{\mathcal{M}}\right),
$$

where $\|\varphi\|^{\mathcal{M}}$ is the truth set of $\varphi$ in $\mathcal{M}$. In the case of finite Scott-Montague frames, the set function $F$ can be seen as a vector-valued Boolean function $f: \mathbb{B}^{n} \rightarrow \mathbb{B}^{n}$, and the Scott-Montague frames thus obtained are called Boolean frames. The idea of Boolean frames was introduced by Virtanen in [19].

It is well known that certain classes of Boolean functions can be defined by means of functional terms (see e.g. [13]). This approach to definability of Boolean function classes has certain similarities to frame definability in modal logic. We achieve

Key words and phrases: Boolean functions, functional terms, term definable classes, clones, propositional logic, modal logic, modal axioms, Kripke frames, Scott-Montague frames.

The work of the first author was partially supported by the Graduate School in Mathematical Logic MALJA. The work of the first and second authors was supported in part by the grant \#28139 from the Academy of Finland. The work of the third author was supported by the grant \#28139 from the Academy of Finland. 
a general correspondence between definability of Boolean functions by functional terms and definability of Scott-Montague frames by modal formulas of a specific form, so-called uniform degree-1 formulas. This correspondence is given by a bijective translation between functional terms and uniform degree- 1 formulas. We prove that a class of Boolean functions is defined by functional terms if and only if the corresponding class of Scott-Montague frames is defined by the uniform degree-1 formulas which are the translations of the defining functional terms. Conversely, a class of Scott-Montague frames is defined by uniform degree- 1 formulas if and only if the corresponding class of Boolean functions is defined by the translations of these formulas.

In this paper we are especially interested in classes of Boolean functions which are closed under composition and contain all projections. These classes are called clones and it is well known that clones are definable by functional terms. As a corollary to our general correspondence result, we obtain for each clone a corresponding class of Scott-Montague frames.

There is a known way to interpret Kripke frames as Scott-Montague frames. Conversely, if a Scott-Montague frame is augmented then it can be interpreted as a Kripke frame. In the case of finite frames, being augmented just means that the frame validates the modal axioms $\square \top$ and $\square(p \wedge q) \leftrightarrow(\square p \wedge \square q)$. For the definition for augmentation, see [3]. This gives rise to the question: What are the connections between classes of Kripke frames and clones. We prove that the class of all Kripke frames corresponds to the clone $\Lambda_{1}$, i.e. to the clone of all conjunctions. Furthermore, by restricting Kripke frames in natural ways, we get classes of Kripke frames corresponding to some subclones of $\Lambda_{1}$.

Not all the classes of Scott-Montague frames corresponding to clones can be characterized by means of the standard Kripke semantics. However, by modifying Kripke semantics, we are also able to characterize the classes of Scott-Montague frames corresponding to the linear clones $L, L_{0}, L_{1}, L_{c}$ and $L S$. The modifications we consider are based on the parity of $R$-successors in Kripke frames. For example, one of the modified semantics is given by

$$
\mathcal{M}, w \models_{O} \square \varphi \text { if and only if }\left|R[w] \cap\|\varphi\|_{O}^{\mathcal{M}}\right| \text { is odd. }
$$

That is, a formula $\square \varphi$ is true at $w$ if and only if $\varphi$ is true at an odd number of $R$-successors of $w$. With respect to this semantics, we show that the class of all Kripke frames corresponds to the clone $L_{0}$, i.e. to the clone of all 0-preserving linear functions. By varying this semantics, we get classes of Kripke frames with given semantics that correspond to the other linear clones.

Structure of the paper. In Section 2, we give the basic definitions and background information on modal logic and Boolean functions. The clones of Boolean functions and the notion of term definability is defined in Section 3. We establish a general correspondence between definability of Boolean functions by functional terms and definability of Scott-Montague frames by uniform degree-1 formulas in Section 4. In Section 5, we present some correspondences between clones and classes 
of Kripke frames, with respect to the standard Kripke semantics. The correspondences between the linear clones and classes of Kripke frames are given in Section 6. In the last section we list some open problems and discuss topics for future work.

Acknowledgement. We are grateful to Ari Virtanen for introducing us to the notion of Boolean frames, and for pointing out the importance of Scott-Montague semantics. The fruitful discussions with Virtanen on his work [19] were the starting point of our research.

\section{Preliminaries}

2.1. Modal logic. Our results concern basic modal logic which is obtained from propositional logic by adding one unary modal operator $\square$. There are several generalizations of this basic modal logic. For example, we could have many different unary modal operators and in that case we are dealing with multi-modal logic. Or we could have modal operators of higher arity. For background and further generalizations, see [1]. Throughout this paper, when we speak of modal logic we mean basic modal logic.

Let $\Phi$ be a countable set of proposition symbols denoted $p_{1}, p_{2}, \ldots$, or $p, q, r, \ldots$. The formulas of modal logic are defined inductively as follows:

(1) All proposition symbols $p \in \Phi$ and constants $\perp$ and $T$ are formulas.

(2) If $\varphi$ and $\psi$ are formulas, then $\neg \varphi,(\varphi \wedge \psi)$ and $\square \varphi$ are formulas.

We also make use of other connectives, like $\vee, \rightarrow$ and $\leftrightarrow$, and define them in a usual way by using $\neg$ and $\wedge$. The dual operator $\diamond$ for $\square$ is defined as $\diamond=\neg \square \neg$. As an analogue to the quantifier rank of a formula in first-order logic we have the concept of the degree of a formula in modal logic. The degree of a formula $\varphi$, denoted by $\operatorname{deg}(\varphi)$, is defined inductively as follows:

$$
\begin{aligned}
\operatorname{deg}(\perp)=\operatorname{deg}(\top) & =0, \\
\operatorname{deg}(p) & =0, \text { for all } p \in \Phi, \\
\operatorname{deg}(\neg \varphi) & =\operatorname{deg}(\varphi), \\
\operatorname{deg}(\varphi \wedge \psi) & =\max \{\operatorname{deg}(\varphi), \operatorname{deg}(\psi)\}, \\
\operatorname{deg}(\square \varphi) & =\operatorname{deg}(\varphi)+1
\end{aligned}
$$

Note that modal formulas of degree 0 are just propositional formulas.

The most popular semantics for modal logic is the relational semantics, known as Kripke semantics. A Kripke frame is a structure $\mathcal{F}=\langle W, R\rangle$ where $W$, called the universe, is a non-empty set and $R$ is a binary relation on $W$. From a Kripke frame we get a Kripke model $\mathcal{M}=\langle\mathcal{F}, V\rangle$ (or $\mathcal{M}=\langle W, R, V\rangle$ ) by adding a valuation function $V: \Phi \rightarrow \mathcal{P}(W)$, where $\mathcal{P}(W)$ denotes the set of all subsets of $W$. The notion of a formula $\varphi$ being true (or satisfied) in the model $\mathcal{M}$ at a state (or world) 
$w \in W$, denoted $\mathcal{M}, w \models \varphi$, is defined inductively as follows:

$$
\begin{aligned}
\mathcal{M}, w=\perp & \text { never, } \\
\mathcal{M}, w=\top & \text { always, } \\
\mathcal{M}, w \models p & \text { iff } w \in V(p), \text { for all } p \in \Phi, \\
\mathcal{M}, w \models \neg \varphi & \text { iff } \mathcal{M}, w \not \models \varphi, \\
\mathcal{M}, w \models \varphi \wedge \psi & \text { iff } \mathcal{M}, w \models \varphi \text { and } \mathcal{M}, w \models \psi, \\
\mathcal{M}, w \models \square \varphi & \text { iff for all } v \in W: \text { if } w R v \text { then } \mathcal{M}, v \models \varphi .
\end{aligned}
$$

It is easy to verify that $\mathcal{M}, w \models \diamond \varphi$ if and only if $\mathcal{M}, v \models \varphi$ for some $v \in W$ such that $w R v$.

In this paper we are also interested in a more general semantics for modal logic, so-called Scott-Montague semantics. A Scott-Montague frame is a structure $\mathcal{F}=$ $\langle W, F\rangle$ where $W$ is a non-empty set and $F$ is a set function $\mathcal{P}(W) \rightarrow \mathcal{P}(W)$. A Scott-Montague model is a structure $\mathcal{M}=\langle\mathcal{F}, V\rangle$ (or $\mathcal{M}=\langle W, F, V\rangle)$ where $\mathcal{F}$ is a Scott-Montague frame and $V$ is a valuation. The truth conditions for the formulas are the same as in Kripke semantics except for the modal operator $\square$, whose interpretation in such a model $\mathcal{M}$ is given by

$$
\mathcal{M}, w=\square \varphi \text { iff } w \in F\left(\|\varphi\|^{\mathcal{M}}\right),
$$

where $\|\varphi\|^{\mathcal{M}}$ denotes the truth set of $\varphi$ in $\mathcal{M}$, i.e. $\|\varphi\|^{\mathcal{M}}=\{v \in W \mid \mathcal{M}, w \models \varphi\}$. In other words $F$ assigns to the truth set of $\varphi$ the truth set of $\square \varphi$, i.e. $\|\square \varphi\|^{\mathcal{M}}=$ $F\left(\|\varphi\|^{\mathcal{M}}\right)$. Sometimes when there is no danger of confusion, we also use a notation $\|\varphi\|$ without a superscript for the truth set of $\varphi$. For an equivalent model theoretic formulation for Scott-Montague semantics, see [3]. Based on that formulation, the semantics is also known as neighbourhood semantics.

Let $\mathcal{M}$ be a Kripke or Scott-Montague model with a universe $W$ and let $\mathcal{F}$ be Kripke or Scott-Montague frame with a universe $W$. We say that a formula $\varphi$ is valid in a model $\mathcal{M}$, denoted $\mathcal{M} \models \varphi$, if $\mathcal{M}, w \models \varphi$ for all $w \in W$. A formula $\varphi$ is valid in a frame $\mathcal{F}$, denoted $\mathcal{F} \models \varphi$, if $\langle\mathcal{F}, V\rangle \models \varphi$ for all $V: \Phi \rightarrow \mathcal{P}(W)$. If $\Psi$ is a set of formulas such that $\mathcal{F}=\varphi$ for all $\varphi \in \Psi$, we use a notation $\mathcal{F} \models \Psi$. Let $\mathcal{C}$ be a class of Kripke frames or a class of Scott-Montague frames and let $\Psi$ be a set of formulas. We say that the class $\mathcal{C}$ is defined by $\Psi$ (or axiomatized by $\Psi$ ) if

$$
\mathcal{F} \in \mathcal{C} \text { iff } \mathcal{F} \models \Psi .
$$

When we talk about definability in modal logic we mean frame-definability since we are interested in sets of formulas defining classes of frames, not classes of models.

Kripke models can be seen as first-order structures. Let $\tau$ be a relational signature with one binary relation symbol $R_{0}$ and unary relation symbols $P_{i}$ for each $p_{i} \in \Phi$. Then each Kripke model $\mathcal{M}=\langle W, R, V\rangle$ can be associated with a $\tau$ structure $\mathcal{M}_{F O}$ with universe $W$ where $R_{0}$ is interpreted as $R$ and each $P_{i}$ is interpreted as $V\left(p_{i}\right)$. Similarly we can interpret Kripke frame $\mathcal{F}=\langle W, R\rangle$ as a $\left\{R_{0}\right\}$-structure $\mathcal{F}_{F O}$. Moreover, it is well known that for all modal formulas $\varphi$ there is a first-order formula $\psi_{\varphi}(x)$ such that for all Kripke models $\mathcal{M}=\langle W, R, V\rangle$ and for all $w \in W$,

$$
\mathcal{M}, w \models \varphi \text { if and only if } \mathcal{M}_{F O} \models \psi_{\varphi}[w] .
$$


The formula $\psi_{\varphi}(x)$ is called the standard translation of $\varphi$. For background, see [1]. In correspondence theory one is interested in knowing when does a modal formula $\varphi$ have a first-order counter-part $\theta_{\varphi}$ such that

$$
\mathcal{F}=\varphi \text { iff } \mathcal{F}_{F O} \models \theta_{\varphi} .
$$

For example, $\mathcal{F} \models \square p \rightarrow p$ if and only if $R$ is reflexive, which is a first-order definable property of $R$. It is well known that from the standard translation of $\varphi$ we get a formula $\theta$ in monadic second-order logic (MSO) such that $\mathcal{F}=\varphi$ iff $\mathcal{F}_{F O} \models \theta$.

In Scott-Montague semantics the validity of a formula $\varphi$ in a frame $\mathcal{F}=\langle W, F\rangle$ corresponds to a set theoretical property of the function $F$. Let $\varphi$ be a formula such that the proposition symbols occurring in $\varphi$ are among $p_{1}, \ldots, p_{k}$. We translate $\varphi$ into a set theoretical expression $S(\varphi)$ inductively as follows:

$$
\begin{aligned}
S\left(p_{i}\right) & =X_{i}, \text { for all } i \in\{1, \ldots, k\}, \\
S(\neg \varphi) & =\overline{S(\varphi)}, \\
S(\varphi \wedge \psi) & =S(\varphi) \cap S(\psi), \\
S(\square \varphi) & =F(S(\varphi)),
\end{aligned}
$$

where we use a notation $\bar{X}$ for the complement of $X \subseteq W$ with respect to $W$. The following proposition is immediate from the translation.

Proposition 1. Let $\mathcal{M}=\langle W, F, V\rangle$ be a Scott-Montague model and let $w \in W$. Let $\varphi$ be a formula with proposition symbols $p_{1}, \ldots, p_{k}$ and let $X_{i}=V\left(p_{i}\right)$ for all $i \in\{1, \ldots, k\}$. Then

$$
\mathcal{M}, w \models \varphi \text { iff } w \in S(\varphi) .
$$

Corollary 1. Let $\mathcal{F}=\langle W, F\rangle$ be a Scott-Montague frame and let $\varphi$ be a formula with proposition symbols $p_{1}, \ldots, p_{k}$. Then

$$
\mathcal{F} \models \varphi \text { iff } S(\varphi)=W \text { for all } X_{1}, \ldots, X_{k} \subseteq W \text {. }
$$

Example. Let $\mathcal{F}=\langle W, F\rangle$ be a Scott-Montague frame and let $\varphi$ be a formula $\square p \rightarrow p$. The formula $\varphi$ is equivalent to a formula $\neg(\square p \wedge \neg p)$. Thus, by Corollary 1 , $\mathcal{F} \models \varphi$ if and only if $\overline{F(X) \cap \bar{X}}=W$ for all $X \subseteq W$. In this case $\varphi$ has only a single occurrence of $\rightarrow$ and we get also a bit more intuitive correspondence result. Namely,

$$
\mathcal{F} \models \square p \rightarrow p \text { iff } F(X) \subseteq X \text { for all } X \subseteq W
$$

2.2. Boolean functions. Let $\mathbb{B}=\{0,1\}$. A Boolean function is a map $f: \mathbb{B}^{n} \rightarrow$ $\mathbb{B}$, for some positive integer $n$ called the arity of $f$. To each sequence $\left(f_{1}, \ldots, f_{m}\right)$ of $n$-ary Boolean functions we associate a Boolean vector-valued map $\left(f_{1}, \ldots, f_{m}\right)$ : $\mathbb{B}^{n} \rightarrow \mathbb{B}^{m}$ defined by $\left(f_{1}, \ldots, f_{m}\right)(\mathbf{a})=\left(f_{1}(\mathbf{a}), \ldots, f_{m}(\mathbf{a})\right)$, for every $\mathbf{a} \in \mathbb{B}^{n}$.

By a class of Boolean functions we simply mean a set $K \subseteq \bigcup_{n \geq 1} \mathbb{B}^{\mathbb{B}^{n}}$, where $\mathbb{B}^{\mathbb{B}^{n}}$ denotes the class of all Boolean functions $f: \mathbb{B}^{n} \rightarrow \mathbb{B}$. In the sequel, we shall say that a vector-valued map $g=\left(f_{1}, \ldots, f_{m}\right)$ belongs to a class $K \subseteq \bigcup_{n \geq 1} \mathbb{B}^{\mathbb{B}^{n}}$ if for every $1 \leq i \leq m$ we have $f_{i} \in K$. 
For each $n \geq 1$, the $n$ different projection maps $\left(a_{1}, \ldots, a_{n}\right) \mapsto a_{i}, 1 \leq i \leq n$, are also called variables, and denoted $x_{1}, \ldots, x_{n}$, where the arity is clear from the context.

Let $f$ be a Boolean function $\mathbb{B}^{n} \rightarrow \mathbb{B}$. For each $1 \leq i \leq n, x_{i}$ is said to be an essential variable of $f$ if there are $a_{1}, \ldots, a_{i-1}, a_{i+1}, \ldots, a_{n}$ in $\mathbb{B}$ such that

$$
f\left(a_{1}, \ldots, a_{i-1}, 0, a_{i+1}, \ldots, a_{n}\right) \neq f\left(a_{1}, \ldots, a_{i-1}, 1, a_{i+1}, \ldots, a_{n}\right) .
$$

Otherwise, $x_{i}$ is called a dummy variable of $f$. Note that constant functions are the only Boolean functions whose variables are all dummy.

The set $\mathbb{B}^{n}$ is a Boolean lattice (distributive and complemented) of $2^{n}$ elements under the component-wise order of vectors given by

$$
\left(a_{1}, \ldots, a_{n}\right) \preceq\left(b_{1}, \ldots, b_{n}\right) \text { if and only if } a_{i} \leq b_{i} \text {, for all } 1 \leq i \leq n \text {. }
$$

The complement of a vector $\mathbf{a}=\left(a_{1}, \ldots, a_{n}\right)$ is also defined component-wise, i.e. $\overline{\mathbf{a}}=\left(1-a_{1}, \ldots, 1-a_{n}\right)$. We denote the all-zero-vector and the all-one-vector by $\mathbf{0}=(0, \ldots, 0)$ and $\mathbf{1}=(1, \ldots, 1)$, respectively. The set $\mathbb{B}^{\mathbb{B}^{n}}$ is also a Boolean lattice of $2^{2^{n}}$ elements under the point-wise ordering of functions given by

$$
f \leq g \text { if and only if } f(\mathbf{a}) \leq g(\mathbf{a}) \text {, for all } \mathbf{a} \in \mathbb{B}^{n} \text {. }
$$

In $\mathbb{B}^{\mathbb{B}^{n}}$, the complement of a Boolean function $f$ is the function $\bar{f}$ defined by $\bar{f}(\mathbf{a})=$ $1-f(\mathbf{a})$, for all $\mathbf{a} \in \mathbb{B}^{n}$. The dual of $f$, denoted $f^{d}$, is given by $f^{d}(\mathbf{a})=\bar{f}(\overline{\mathbf{a}})$, for all $\mathbf{a} \in \mathbb{B}^{n}$. The functions (of any arity) having constant value 0 and 1 on $\mathbb{B}^{n}$ are denoted by $\mathbf{0}$ and $\mathbf{1}$, respectively.

Boolean functions are many times defined as maps which can be "specified" by means of "Boolean formulas" (see e.g. [2]). Let $X$ be a countable set of vectorvariable symbols $\mathbf{x}_{1}, \mathbf{x}_{2}, \ldots$ A Boolean formula is a formal expression defined inductively as follows:

(1) All vector-variable symbols $\mathbf{x}_{i} \in X$ and constant functions $\mathbf{0}$ and $\mathbf{1}$ are Boolean formulas.

(2) If $G_{1}$ and $G_{2}$ are Boolean formulas, then $\neg G_{1}$ and $\left(G_{1} \wedge G_{2}\right)$ are Boolean formulas.

We also make use of other connectives, like $\vee, \rightarrow$ and $\leftrightarrow$, as usually defined. We write $G\left(\mathbf{x}_{1}, \ldots, \mathbf{x}_{n}\right)$ if the vector-variable symbols are among $\mathbf{x}_{1}, \ldots, \mathbf{x}_{n}$, and we say that $G$ has arity $n$.

Each Boolean formula $G$ specifies a Boolean function $f$ by interpreting the expression $G$ in the Boolean lattice $\mathbb{B}$, and in this case $G$ is said to be a formal represention of $f$. It is well known that every Boolean function $f$ can be represented by a DNF (disjunctive normal form), i.e. by an expression of the form

$$
\bigvee_{i \in I}\left(\bigwedge_{j \in P_{i}} x_{j} \wedge \bigwedge_{j \in N_{i}} \bar{x}_{j}\right)
$$

where $I$ is a finite set of indices, and in each conjunct each variable appears at most once. We regard empty disjunctions and empty conjunctions as having value 0 and 
1 , respectively. It is easy to verify that if

$$
f=\bigvee_{i \in I}\left(\bigwedge_{j \in P_{i}} x_{j} \wedge \bigwedge_{j \in N_{i}} \bar{x}_{j}\right)
$$

then the dual $f^{d}$ of $f$ is represented by

$$
f^{d}=\bigwedge_{i \in I}\left(\bigvee_{j \in P_{i}} x_{j} \vee \bigvee_{j \in N_{i}} \bar{x}_{j}\right)
$$

Expressions of the form (1) are called CNF (conjunctive normal form) representations. Note that DNF and CNF representations are not unique.

Since Stone [17], it is well-known that any Boolean lattice can be viewed as a Boolean ring (in which every element is idempotent under product) by defining multiplication and addition $(\bmod 2)$ by

$$
x \cdot y=x \wedge y \text { and } x \oplus y=(\bar{x} \wedge y) \vee(x \wedge \bar{y}) .
$$

Thus both $\mathbb{B}^{n}$ and $\mathbb{B}^{\mathbb{B}^{n}}$ can also be treated as Boolean rings using the above algebraic translations. It is not difficult to see that each $n$-ary Boolean function $f$ can be represented in this Boolean ring language by a multilinear polynomial in $n$ indeterminates over $\mathbb{B}$, called its Zhegalkin polynomial or Reed-Muller polynomial

$$
f=\Sigma_{j \in I}\left(c_{j} \cdot \prod_{i \in I_{j}} x_{i}\right)
$$

Moreover, unlike DNF and CNF representations, the Zhegalkin polynomial representation of a Boolean function is unique (up to permutation of terms and permutation of variables in the terms). For further developments on Boolean normal forms, see [6].

If $f$ is an $n$-ary Boolean function and $g_{1}, \ldots, g_{n}$ are $m$-ary Boolean functions, then their composition is the $m$-ary Boolean function $f\left(g_{1}, \ldots, g_{n}\right)$, whose value on every $\mathbf{a} \in \mathbb{B}^{m}$ is $f\left(g_{1}(\mathbf{a}), \ldots, g_{n}(\mathbf{a})\right)$. Let $I$ and $J$ be classes of Boolean functions of various arities. The class composition $I \circ J$, is defined as the set of all composites of functions in $I$ with functions in $J$, i.e.

$$
I \circ J=\left\{f\left(g_{1}, \ldots, g_{n}\right) \mid n, m \geq 1, f n \text {-ary in } I, g_{1}, \ldots, g_{n} m \text {-ary in } J\right\} .
$$

When $I=\{f\}$, we write $f \circ J$ instead of $\{f\} \circ J$. (For background see [4] and [5].)

\section{Clones and Term definable classes}

A (Boolean) clone is a class $C \subseteq \bigcup_{n \geq 1} \mathbb{B}^{\mathbb{B}^{n}}$ containing all projections and idempotent under class composition, that is, satisfying $C \circ C=C$. The clones of Boolean functions form an algebraic lattice by defining the meet as the intersection of clones, and the join as the smallest clone containing the union. This lattice is known as Post Lattice (see Figure 1), named after Emil Post who first described and classified in [14] the set of all Boolean clones (for recent and shorter proofs of Post's classification see [15], [18], [20]; for general background see [10] and [11]).

In this paper we shall be especially interested in the following clones:

- $\Omega$ : the class of all Boolean functions; 


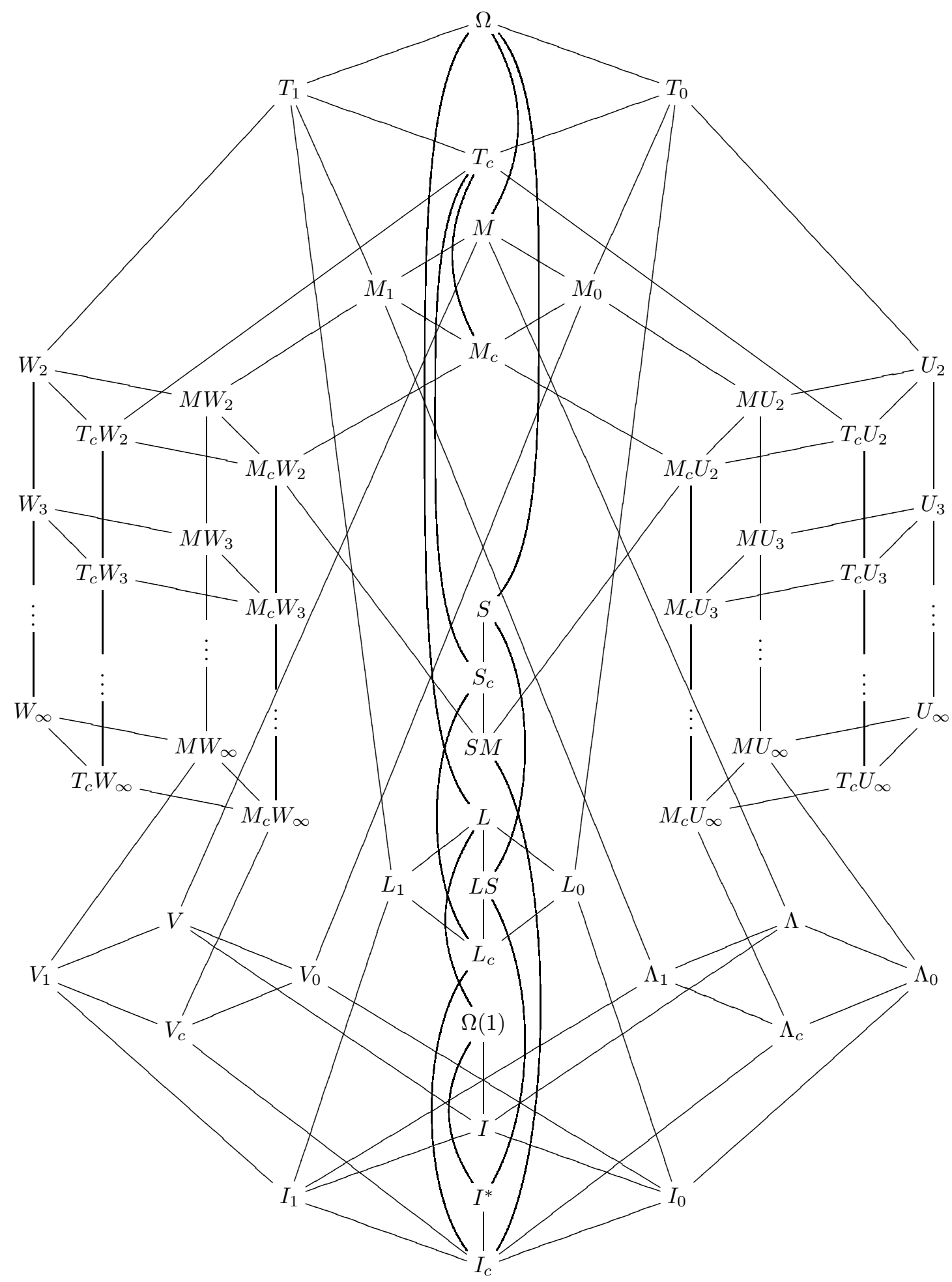

Figure 1. Post Lattice. 
- $T_{0}$ and $T_{1}$ : the classes of 0 - and 1-preserving functions, respectively, i.e., $T_{0}=\{f \in \Omega \mid f(0, \ldots, 0)=0\}, T_{1}=\{f \in \Omega \mid f(1, \ldots, 1)=1\} ;$

- $T_{c}$ : the class of constant-preserving functions, i.e., $T_{c}=T_{0} \cap T_{1}$.

- $M$ : the class of all monotone functions, i.e., $M=\{f \in \Omega \mid f(\mathbf{a}) \leq f(\mathbf{b})$, whenever $\mathbf{a} \preceq \mathbf{b}\}$

- $S$ : the class of all self-dual functions, i.e., $S=\left\{f \in \Omega \mid f^{d}=f\right\}$

- $S_{c}=S \cap T_{c}, S M=S \cap M$;

- $L$ : the class of all linear functions, i.e., $L=\left\{f \in \Omega \mid f=c_{0} \mathbf{1} \oplus c_{1} x_{1} \oplus \cdots \oplus c_{n} x_{n}\right.$ for some $n$ and $\left.c_{0}, \ldots, c_{n} \in \mathbb{B}\right\} ;$

- $L_{0}=L \cap T_{0}, L_{1}=L \cap T_{1}, L S=L \cap S, L_{c}=L \cap T_{c}$;

- $\Lambda$ : the class of all conjunctions and constants, i.e., $\Lambda=\left\{f \in \Omega \mid f=\mathbf{0}, \mathbf{1}, x_{i_{1}} \wedge \cdots \wedge x_{i_{n}}\right.$ for some $n \geq 1$ and $i_{j}$ 's $\}$

- $\Lambda_{0}=\Lambda \cap T_{0}, \Lambda_{1}=\Lambda \cap T_{1}, \Lambda_{c}=\Lambda \cap T_{c}$;

- $V$ : the class of all disjunctions and constants, i.e., $V=\left\{f \in \Omega \mid f=\mathbf{0}, \mathbf{1}, x_{i_{1}} \vee \cdots \vee x_{i_{n}}\right.$ for some $n \geq 1$ and $\left.i_{j}^{\prime} s\right\}$

- $V_{0}=V \cap T_{0}, V_{1}=V \cap T_{1}, V_{c}=V \cap T_{c}$;

- $\Omega(1)$ : the class of all variables, negated variables, and constants;

- $I^{*}$ : the class of all variables and negated variables;

- $I$ : the class of all variables and constants;

- $I_{0}=I \cap T_{0}, I_{1}=I \cap T_{1}$;

- $I_{c}$ : the smallest clone containing only variables, i.e., $I_{c}=I \cap T_{c}$.

Remark 1. Using the above definitions, it is easy to verify that every function in:

- $L_{0}$ is of the form $f=c\left(x_{1} \oplus \cdots \oplus x_{n}\right)$, for $n \geq 1$ and $c \in \mathbb{B}$,

- $L_{1}$ is either the sum of an odd number of variables, or the negation of the sum of an even number of variables,

- $L S$ is either the sum of an odd number of variables, or the negation of the sum of an odd number of variables,

- $L_{c}$ is the sum of an odd number of variables.

Observe also that the only functions in $\Lambda$ with essential variables are conjunctions of variables, and since $\mathbf{0} \notin \Lambda_{1}$, for every $f \in \mathbb{B}^{\mathbb{B}^{n}} \cap \Lambda_{1}$, we have that

$f\left(a_{1}, \ldots, a_{n}\right)=1$ if and only if $a_{i}=1$, whenever $x_{i}$ is an essential variable of $f$.

A functional term is a formal expression

$$
T=G\left(\mathbf{f}\left(G_{1}\left(\mathbf{x}_{1}, \ldots, \mathbf{x}_{r}\right)\right), \ldots, \mathbf{f}\left(G_{m}\left(\mathbf{x}_{1}, \ldots, \mathbf{x}_{r}\right)\right)\right)
$$

where $m, r \geq 1, G$ is an $m$-ary Boolean formula, $G_{1}, \ldots, G_{m}$ are $r$-ary Boolean formulas, $\mathbf{x}_{1}, \ldots, \mathbf{x}_{r}$ are $r$ distinct vector-variable symbols, and $\mathbf{f}$ is a function symbol. Note that, for each positive integer $n$, the functional term $T$ defines a map $T: \mathbb{B}^{\mathbb{B}^{n}} \times \mathbb{B}^{n r} \rightarrow \mathbb{B}$ given by

$$
T\left(f, \mathbf{a}_{1}, \ldots, \mathbf{a}_{r}\right)=G\left(f\left(G_{1}\left(\mathbf{a}_{1}, \ldots, \mathbf{a}_{r}\right)\right), \ldots, f\left(G_{m}\left(\mathbf{a}_{1}, \ldots, \mathbf{a}_{r}\right)\right)\right)
$$

by interpreting the Boolean formula $G$ in $\mathbb{B}$ and the Boolean formulas $G_{i}$ in $\mathbb{B}^{n}$. 
An $n$-ary Boolean function $f$ is said to satisfy the functional term $T$, denoted $T(f) \equiv 1$, if for all $\mathbf{a}_{1}, \ldots, \mathbf{a}_{r} \in \mathbb{B}^{n}$ we have

$$
T\left(f, \mathbf{a}_{1}, \ldots, \mathbf{a}_{r}\right)=1
$$

For background, see $[13,8]$. See also $[7,12]$ for further variants, and [5] for further extensions. For our purposes, we extend this notion of satisfaction to Boolean vector-valued maps $g=\left(f_{1}, \ldots, f_{n}\right)$, by saying that $g$ satisfies (2) if for each $1 \leq$ $i \leq n, f_{i}$ satisfies (2). In this setting, a class $K \subseteq \bigcup_{n>1} \mathbb{B}^{\mathbb{B}^{n}}$ is said to be defined by a set $\mathcal{T}$ of functional terms, denoted $K=\langle\mathcal{T}\rangle$, if $K$ is the set of all those functions satisfying every member of $\mathcal{T}$. If $\mathcal{T}=\{T\}$, then we write $K=\langle T\rangle$ instead of $K=\langle\{T\}\rangle$. A class $K$ is said to be term definable if there is a set $\mathcal{T}$ of functional terms such that $K=\langle\mathcal{T}\rangle$.

An $m$-ary Boolean function $g$ is said to be obtained from an $n$-ary Boolean function $f$ by simple variable substitution, if there are $m$-ary projections $p_{1}, \ldots, p_{n} \in$ $I_{c}$ such that $g=f\left(p_{1}, \ldots, p_{n}\right)$. A class $K \subseteq \Omega$ is said to be closed under simple variable substitutions if each Boolean function obtained from a function $f$ in $K$ by simple variable substitution is also in $K$. In other words, the class $K$ is closed under simple variable substitutions if and only if $K \circ I_{c}=K$. See [4, 5] for generalizations of the notion of simple variable substitution. The relevance of simple variable substitutions for definability of Boolean function classes by means of functional terms is made apparent by following result which was first obtained, in a somewhat variant form, by Ekin, Foldes, Hammer and Hellerstein in [7].

Theorem 1. A class $K$ of Boolean functions is definable by a set of functional terms if and only if $K$ is closed under simple variable substitutions.

Note that every clone is in particular closed under simple variable substitutions, and thus every clone is definable by some set functional terms. In the sequel, we shall make use of some of the examples listed below. For a complete list of functional terms defining every clone of Boolean functions, see [8].

$$
\begin{aligned}
& T_{0}=\langle\neg \mathbf{f}(\mathbf{0})\rangle, \\
& T_{1}=\langle\mathbf{f}(\mathbf{1})\rangle, \\
& T_{c}=\langle\{\neg \mathbf{f}(\mathbf{0}), \mathbf{f}(\mathbf{1})\}\rangle, \\
& M=\left\langle\mathbf{f}\left(\mathbf{x}_{1}\right) \rightarrow \mathbf{f}\left(\mathbf{x}_{2} \vee \mathbf{x}_{1}\right)\right\rangle \\
& S=\left\langle\neg \mathbf{f}\left(\mathbf{x}_{1}\right) \leftrightarrow \mathbf{f}\left(\neg \mathbf{x}_{1}\right)\right\rangle \\
& L=\left\langle\mathbf{1} \oplus \mathbf{f}(\mathbf{0}) \oplus \mathbf{f}\left(\mathbf{x}_{1} \oplus \mathbf{x}_{2}\right) \oplus \mathbf{f}\left(\mathbf{x}_{1}\right) \oplus \mathbf{f}\left(\mathbf{x}_{2}\right)\right\rangle \\
& L_{0}=\left\langle\mathbf{1} \oplus \mathbf{f}\left(\mathbf{x}_{1} \oplus \mathbf{x}_{2}\right) \oplus \mathbf{f}\left(\mathbf{x}_{1}\right) \oplus \mathbf{f}\left(\mathbf{x}_{2}\right)\right\rangle \\
& L_{1}=\left\langle\left\{\mathbf{f}(\mathbf{1}), \mathbf{1} \oplus \mathbf{f}(\mathbf{0}) \oplus \mathbf{f}\left(\mathbf{x}_{1} \oplus \mathbf{x}_{2}\right) \oplus \mathbf{f}\left(\mathbf{x}_{1}\right) \oplus \mathbf{f}\left(\mathbf{x}_{2}\right)\right\}\right\rangle \\
& L S=\left\langle\left\{\mathbf{f}(\mathbf{1}) \oplus \mathbf{f}(\mathbf{0}), \mathbf{1} \oplus \mathbf{f}(\mathbf{0}) \oplus \mathbf{f}\left(\mathbf{x}_{1} \oplus \mathbf{x}_{2}\right) \oplus \mathbf{f}\left(\mathbf{x}_{1}\right) \oplus \mathbf{f}\left(\mathbf{x}_{2}\right)\right\}\right\rangle \\
& L_{c}=\left\langle\left\{\mathbf{f}(\mathbf{1}), \mathbf{1} \oplus \mathbf{f}\left(\mathbf{x}_{1} \oplus \mathbf{x}_{2}\right) \oplus \mathbf{f}\left(\mathbf{x}_{1}\right) \oplus \mathbf{f}\left(\mathbf{x}_{2}\right)\right\}\right\rangle \\
& \Lambda=\left\langle\mathbf{f}\left(\mathbf{x}_{1} \wedge \mathbf{x}_{2}\right) \leftrightarrow \mathbf{f}\left(\mathbf{x}_{1}\right) \wedge \mathbf{f}\left(\mathbf{x}_{2}\right)\right\rangle \\
& \Lambda_{1}=\left\langle\left\{\mathbf{f}(\mathbf{1}), \mathbf{f}\left(\mathbf{x}_{1} \wedge \mathbf{x}_{2}\right) \leftrightarrow \mathbf{f}\left(\mathbf{x}_{1}\right) \wedge \mathbf{f}\left(\mathbf{x}_{2}\right)\right\}\right\rangle \\
& \Lambda_{c}=\left\langle\left\{\neg \mathbf{f}(\mathbf{0}), \mathbf{f}(\mathbf{1}), \mathbf{f}\left(\mathbf{x}_{1} \wedge \mathbf{x}_{2}\right) \leftrightarrow \mathbf{f}\left(\mathbf{x}_{1}\right) \wedge \mathbf{f}\left(\mathbf{x}_{2}\right)\right\}\right\rangle, \\
& I=\left\langle\left\{\mathbf{f}\left(\mathbf{x}_{1} \wedge \mathbf{x}_{2}\right) \leftrightarrow \mathbf{f}\left(\mathbf{x}_{1}\right) \wedge \mathbf{f}\left(\mathbf{x}_{2}\right), \mathbf{f}\left(\mathbf{x}_{1} \vee \mathbf{x}_{2}\right) \leftrightarrow \mathbf{f}\left(\mathbf{x}_{1}\right) \vee \mathbf{f}\left(\mathbf{x}_{2}\right)\right\}\right\rangle, \\
& I_{1}=\left\langle\left\{\mathbf{f}(\mathbf{1}), \mathbf{f}\left(\mathbf{x}_{1} \wedge \mathbf{x}_{2}\right) \leftrightarrow \mathbf{f}\left(\mathbf{x}_{1}\right) \wedge \mathbf{f}\left(\mathbf{x}_{2}\right), \neg \mathbf{f}\left(\mathbf{x}_{1}\right) \rightarrow \mathbf{f}\left(\neg \mathbf{x}_{1}\right)\right\}\right\rangle \\
& I_{c}=\left\langle\left\{\mathbf{f}(\mathbf{1}), \mathbf{f}\left(\mathbf{x}_{1} \wedge \mathbf{x}_{2}\right) \leftrightarrow \mathbf{f}\left(\mathbf{x}_{1}\right) \wedge \mathbf{f}\left(\mathbf{x}_{2}\right), \neg \mathbf{f}\left(\mathbf{x}_{1}\right) \leftrightarrow \mathbf{f}\left(\neg \mathbf{x}_{1}\right)\right\}\right\rangle .
\end{aligned}
$$


Note that if a class $K$ is defined by a finite set $\mathcal{T}$ of functional terms, then it is defined by a single functional term which is obtained by taking the conjunction of the functional terms in $\mathcal{T}$. For example,

$$
\Lambda_{1}=\left\langle\mathbf{f}(\mathbf{1}) \wedge\left(\mathbf{f}\left(\mathbf{x}_{1} \wedge \mathbf{x}_{2}\right) \leftrightarrow \mathbf{f}\left(\mathbf{x}_{1}\right) \wedge \mathbf{f}\left(\mathbf{x}_{2}\right)\right)\right\rangle .
$$

\section{General correspondence}

In this section we will show that definability of Boolean functions by functional terms corresponds exactly to definability of finite Scott-Montague frames by formulas of a specific form. We call these formulas uniform degree-1 formulas. We will make use of the natural bijection between vector-valued Boolean functions $f: \mathbb{B}^{n} \rightarrow \mathbb{B}^{n}$ and set functions $F: \mathcal{P}(W) \rightarrow \mathcal{P}(W)$, where $W$ is a set with $n$ elements. By using this natural bijection, finite Scott-Montague frames can be interpreted by Boolean functions and this correspondence can be used to define certain classes of frames by using known theories of Boolean function classes. We

will only consider finite frames and throughout this section we fix $W$ to be the $n$-element set $\left\{w_{1}, \ldots, w_{n}\right\}$.

Let $X \subseteq W$ and $\mathbf{x} \in \mathbb{B}^{n}$. By $\mathbf{a}_{X}$ we denote the characteristic vector of $X$, i.e. for all $1 \leq i \leq n$,

$$
\mathbf{a}_{X}[i]=1 \text { if and only if } w_{i} \in X,
$$

where $\mathbf{a}_{X}[i]$ denotes the $i$ th component of the vector $\mathbf{a}_{X}$. Conversely, by $A_{\mathbf{x}}$ we denote the set whose characteristic vector is $\mathbf{x}$. It is immediate that $\mathbf{a}_{A_{\mathbf{x}}}=\mathbf{x}$ and $A_{\mathbf{a}_{X}}=X$.

Let $f=\left(f_{1}, \ldots, f_{n}\right)$ be a vector-valued Boolean function $\mathbb{B}^{n} \rightarrow \mathbb{B}^{n}$. The functions $f_{i}$ are called the component functions, or simply the components, of $f$. We define $F_{f}$ to be the function $\mathcal{P}(W) \rightarrow \mathcal{P}(W)$ such that $F_{f}(X)=A_{f\left(\mathbf{a}_{X}\right)}$ for all $X \subseteq W$. That is, for all $X \subseteq W$ and $w_{i} \in W$,

$$
w_{i} \in F_{f}(X) \text { if and only if } f_{i}\left(\mathbf{a}_{X}\right)=1 .
$$

We denote by $\mathcal{F}_{f}$ the Scott-Montague frame $\left\langle W, F_{f}\right\rangle$.

Let $\mathcal{F}=\langle W, F\rangle$ be a Scott-Montague frame. We denote by $f_{F}$ the vector-valued Boolean function $\mathbb{B}^{n} \rightarrow \mathbb{B}^{n}$ for which $f_{F}(\mathbf{x})=\mathbf{a}_{F\left(A_{\mathbf{x}}\right)}$ for all $\mathbf{x} \in \mathbb{B}^{n}$. In other words, for all $\mathbf{x} \in \mathbb{B}^{n}$ and for all $1 \leq i \leq n$,

$$
f_{i}(\mathbf{x})=1 \text { if and only if } w_{i} \in F\left(A_{\mathbf{x}}\right),
$$

where $f_{i}$ is the $i$ th component of $f_{F}$. We also use a notation $f_{\mathcal{F}}=f_{F}$.

It is immediate from these definitions that $f_{F_{f}}=f$ and $F_{f_{F}}=F$.

Let $T$ be a functional term, let $f=\left(f_{1}, \ldots, f_{n}\right)$ be a vector-valued Boolean function $\mathbb{B}^{n} \rightarrow \mathbb{B}^{n}$ and let $\mathbf{a}_{1}, \ldots, \mathbf{a}_{r} \in \mathbb{B}^{n}$. By $T\left(f, \mathbf{a}_{1}, \ldots, \mathbf{a}_{r}\right)$ we denote the $n$-vector whose $i$ th component is $T\left(f_{i}, \mathbf{a}_{1}, \ldots, \mathbf{a}_{r}\right)$. We write $T(f) \equiv \mathbf{1}$ when $T\left(f, \mathbf{a}_{1}, \ldots, \mathbf{a}_{r}\right)=\mathbf{1}$ for all $\mathbf{a}_{1}, \ldots, \mathbf{a}_{r} \in \mathbb{B}^{n}$.

In order to establish the desired connection between term definable classes of Boolean functions and definable classes of Scott-Montague frames, we need a translation of functional terms into modal formulas and, conversely, of modal formulas 
into functional terms. We will first define a translation of functional terms $T$ into modal formulas $\psi_{T}$ such that for every vector-valued Boolean function $f, T(f) \equiv \mathbf{1}$ if and only if $\mathcal{F}_{f} \models \psi_{T}$. The idea of the translation is very simple; we just replace vector-variable symbols $\mathbf{x}_{i}$ by proposition symbols $p_{i}$ and the function symbol $\mathbf{f}$ by the modal operator $\square$, and all the connectives remain the same. The exact definition of the translation is done in two parts. First we define a translation for Boolean formulas and then, using this, we define a translation for functional terms.

Let $G=G\left(\mathbf{x}_{1}, \ldots, \mathbf{x}_{r}\right)$ be an $r$-ary Boolean formula. The translation of $G$, denoted by $\psi_{G}$, is defined inductively as follows.

- If $G=\mathbf{0}$ (or $\mathbf{1}$ ), then $\psi_{G}=\perp$ (or $\top$ respectively).

- If $G\left(\mathbf{x}_{1}, \ldots, \mathbf{x}_{r}\right)=\mathbf{x}_{i}$ for some $1 \leq i \leq r$, then $\psi_{G}=p_{i}$.

- If $G=\neg H$, then $\psi_{G}=\neg \psi_{H}$.

- If $G=H_{1} \wedge H_{2}$, then $\psi_{G}=\psi_{H_{1}} \wedge \psi_{H_{2}}$.

Let $T=G\left(\mathbf{f}\left(G_{1}\left(\mathbf{x}_{1}, \ldots, \mathbf{x}_{r}\right)\right), \ldots, \mathbf{f}\left(G_{m}\left(\mathbf{x}_{1}, \ldots, \mathbf{x}_{r}\right)\right)\right)$ be a functional term as defined in Section 3 , let $\psi_{G}\left(p_{1}, \ldots, p_{m}\right)$ be the translation of $G$ and let $\psi_{G_{i}}$ be the translation of $G_{i}$ for each $1 \leq i \leq m$. The translation of $T$, denoted by $\psi_{T}$, is defined as

$$
\psi_{T}=\psi_{G}\left(\square \psi_{G_{1}}, \ldots, \square \psi_{G_{m}}\right) .
$$

For example, let $T=\mathbf{f}\left(\mathbf{x}_{1} \wedge \mathbf{x}_{2}\right) \rightarrow\left(\mathbf{f}\left(\mathbf{x}_{1}\right) \wedge \mathbf{f}\left(\mathbf{x}_{2}\right)\right)$. Then

$$
\psi_{T}=\square\left(p_{1} \wedge p_{2}\right) \rightarrow\left(\square p_{1} \wedge \square p_{2}\right) .
$$

Let $\mathbf{a}=\left(\mathbf{a}_{1}, \ldots, \mathbf{a}_{r}\right) \in \mathbb{B}^{n r}$. We denote by $V_{\mathbf{a}}$ the valuation function $\Phi \rightarrow \mathcal{P}(W)$ such that $V_{\mathbf{a}}\left(p_{j}\right)=A_{\mathbf{a}_{j}}$ for all $1 \leq j \leq r$.

Lemma 1. Let $G$ be an $r$-ary Boolean formula and let $\mathbf{a}=\left(\mathbf{a}_{1}, \ldots, \mathbf{a}_{r}\right) \in \mathbb{B}^{n r}$. Let $\mathcal{M}=\left\langle W, F, V_{\mathbf{a}}\right\rangle$ be a Scott-Montague model. Then for all $1 \leq i \leq n$,

$$
G\left(\mathbf{a}_{1}, \ldots, \mathbf{a}_{r}\right)[i]=1 \text { if and only if } \mathcal{M}, w_{i}=\psi_{G},
$$

i.e. $\left\|\psi_{G}\right\|^{\mathcal{M}}=A_{\mathbf{b}}$, where $\mathbf{b}=G\left(\mathbf{a}_{1}, \ldots, \mathbf{a}_{r}\right)$.

Proof. The proof is by induction on the construction of $G$. If $G$ is a constant $\mathbf{0}$ (or 1) then $\psi_{G}$ is $\perp$ (or $\top$ respectively) and the result is trivial. If $G\left(\mathbf{x}_{1}, \ldots, \mathbf{x}_{r}\right)=\mathbf{x}_{j}$ for some $1 \leq j \leq r$ then $\psi_{G}=p_{j}$ and

$$
G\left(\mathbf{a}_{1}, \ldots, \mathbf{a}_{r}\right)[i]=1 \Leftrightarrow \mathbf{a}_{j}[i]=1 \Leftrightarrow w_{i} \in A_{\mathbf{a}_{j}} \Leftrightarrow \mathcal{M}, w_{i} \models p_{j} \Leftrightarrow \mathcal{M}, w_{i} \models \psi_{G} .
$$

Let $G$ be of the form $\neg H$ and suppose that the claim holds for $H$. Then

$$
\begin{aligned}
& G\left(\mathbf{a}_{1}, \ldots, \mathbf{a}_{r}\right)[i]=1 \quad \Leftrightarrow H\left(\mathbf{a}_{1}, \ldots, \mathbf{a}_{r}\right)[i]=0 \\
& \stackrel{*}{\Leftrightarrow} \mathcal{M}, w_{i} \not \neq \psi_{H} \\
& \Leftrightarrow \mathcal{M}, w_{i} \models \neg \psi_{H} \\
& \Leftrightarrow \mathcal{M}, w_{i} \models \psi_{G},
\end{aligned}
$$

where the equivalence $(*)$ holds by the induction hypothesis. In the case where $G$ is of the form $H_{1} \wedge H_{2}$, the result also follows straightforwardly from the induction hypothesis. 
Theorem 2. Let $T$ be a functional term

$$
G\left(\mathbf{f}\left(G_{1}\left(\mathbf{x}_{1}, \ldots, \mathbf{x}_{r}\right), \ldots, \mathbf{f}\left(G_{m}\left(\mathbf{x}_{1}, \ldots, \mathbf{x}_{r}\right)\right)\right)\right.
$$

and let $\mathbf{a}=\left(\mathbf{a}_{1}, \ldots, \mathbf{a}_{r}\right) \in \mathbb{B}^{n r}$. Let $f$ be a vector-valued Boolean function $\mathbb{B}^{n} \rightarrow \mathbb{B}^{n}$. Then for all $1 \leq i \leq n$,

$$
T\left(f, \mathbf{a}_{1}, \ldots, \mathbf{a}_{r}\right)[i]=1 \text { if and only if }\left\langle\mathcal{F}_{f}, V_{\mathbf{a}}\right\rangle, w_{i}=\psi_{T} .
$$

Proof. The proof is by induction on the construction of $G$. The cases where $G$ is a constant, or of the form $\neg H$ or $H_{1} \wedge H_{2}$, are handled similarly as in the previous lemma. If $G\left(\mathbf{x}_{1}, \ldots, \mathbf{x}_{m}\right)=\mathbf{x}_{j}$ for some $1 \leq j \leq m$, then $T=\mathbf{f}\left(G_{j}\left(\mathbf{x}_{1}, \ldots, \mathbf{x}_{r}\right)\right)$ and $\psi_{T}=\square \psi_{G_{j}}$. Thus

$$
\begin{aligned}
T\left(f, \mathbf{a}_{1}, \ldots, \mathbf{a}_{r}\right)[i]=1 & \Leftrightarrow f\left(G_{j}\left(\mathbf{a}_{1}, \ldots, \mathbf{a}_{r}\right)\right)[i]=1 \\
& \Leftrightarrow w_{i} \in A_{f}(\mathbf{b}), \text { where } \mathbf{b}=G_{j}\left(\mathbf{a}_{1}, \ldots, \mathbf{a}_{r}\right) \\
& \Leftrightarrow w_{i} \in F_{f}\left(A_{\mathbf{b}}\right), \text { where } \mathbf{b}=G_{j}\left(\mathbf{a}_{1}, \ldots, \mathbf{a}_{r}\right) \\
& \stackrel{*}{\Leftrightarrow} w_{i} \in F_{f}\left(\left\|\psi_{G_{j}}\right\| \mathcal{M}\right), \text { where } \mathcal{M}=\left\langle\mathcal{F}_{f}, V_{\mathbf{a}}\right\rangle \\
& \Leftrightarrow\left\langle\mathcal{F}_{f}, V_{\mathbf{a}}\right\rangle, w_{i}=\square \psi_{G_{j}} \\
& \Leftrightarrow\left\langle\mathcal{F}_{f}, V_{\mathbf{a}}\right\rangle, w_{i} \models \psi_{T},
\end{aligned}
$$

where the equivalence $(*)$ holds by Lemma 1 .

As an immediate corollary to Theorem 2 we get that the translation $\psi_{T}$ works as desired.

Corollary 2. Let $T$ be a functional term and let $\mathbf{a}=\left(\mathbf{a}_{1}, \ldots, \mathbf{a}_{r}\right) \in \mathbb{B}^{n r}$. Let $f$ be a vector-valued Boolean function $\mathbb{B}^{n} \rightarrow \mathbb{B}^{n}$. Then

$$
T\left(f, \mathbf{a}_{1}, \ldots, \mathbf{a}_{r}\right)=\mathbf{1} \text { if and only if }\left\langle\mathcal{F}_{f}, V_{\mathbf{a}}\right\rangle \models \psi_{T},
$$

and futhermore

$$
T(f) \equiv \mathbf{1} \text { if and only if } \mathcal{F}_{f} \models \psi_{T} .
$$

The translation of a functional term $T$ yields a modal formula of a specific form. We say that $\psi$ is a uniform degree-1 formula if it is of the form

$$
\psi=\varphi\left(\square \varphi_{1}, \ldots, \square \varphi_{m}\right),
$$

where $\varphi, \varphi_{1}, \ldots, \varphi_{m}$ are propositional formulas. Here $\varphi$ is a formula with proposition symbols $p_{1}, \ldots, p_{m}$ and $\psi$ is a formula obtained from $\varphi$ by replacing every occurrence of $p_{i}$ by $\square \varphi_{i}$ for each $1 \leq i \leq m$. Thus $\psi$ is of degree 1 and uniform in the sense that $\psi$ has no subformula in which a binary Boolean connective combines a formula of the form $\square \theta$ and a propositional formula other than a constant. For example, $T \wedge \square \top \wedge \square p_{1}$ is a uniform degree- 1 formula, whereas $\square p_{1} \vee p_{2}$ is not.

Our next aim is to translate uniform degree- 1 formulas $\psi$ into functional terms $T_{\psi}$ such that for every Scott-Montague frame $\mathcal{F}, \mathcal{F} \models \psi$ if and only if $T_{\psi}\left(f_{\mathcal{F}}\right) \equiv \mathbf{1}$. The idea of the translation is similar to the one introduced above. We just replace the modal operator $\square$ in $\psi$ by the function symbol $\mathbf{f}$ and proposition symbols $p_{i}$ by vector-variable symbols $\mathbf{x}_{i}$. As we will observe later in Theorem 4 , the translations $T \mapsto \psi_{T}$ and $\psi \mapsto T_{\psi}$ are inverses of each other. We will first define a translation 
of a propositional formula $\varphi$ into a Boolean formula $G_{\varphi}$, and then using this we define a translation of a uniform degree-1 formula into a functional term.

If $\varphi$ is a modal formula in which there occur $r$ different proposition symbols, we always assume for simplicity that those proposition symbols are $p_{1}, \ldots, p_{r}$. Let $\varphi$ be a propositional formula. The translation of $\varphi$, denoted by $G_{\varphi}$, is defined inductively as follows.

- If $\varphi=\perp$ (or $\top$ ), then $G_{\varphi}=\mathbf{0}$ (or $\mathbf{1}$ respectively).

- If $\varphi\left(p_{1}, \ldots, p_{r}\right)=p_{i}$ for some $1 \leq i \leq r$, then $G_{\varphi}\left(\mathbf{x}_{1}, \ldots, \mathbf{x}_{r}\right)=\mathbf{x}_{i}$.

- If $\varphi=\neg \theta$, then $G_{\varphi}=\neg G_{\theta}$.

- If $\varphi=\theta_{1} \wedge \theta_{2}$, then $G_{\varphi}=G_{\theta_{1}} \wedge G_{\theta_{2}}$.

Let $\psi=\varphi\left(\square \varphi_{1}, \ldots, \square \varphi_{m}\right)$ be a uniform degree-1 formula. The translation of $\psi$, denoted by $T_{\psi}$, is defined as

$$
T_{\psi}=G_{\varphi}\left(\mathbf{f}\left(G_{\varphi_{1}}\left(\mathbf{x}_{1}, \ldots, \mathbf{x}_{r}\right)\right), \ldots, \mathbf{f}\left(G_{\varphi_{m}}\left(\mathbf{x}_{1}, \ldots, \mathbf{x}_{r}\right)\right)\right) .
$$

If, for example, $\psi=\square \top \wedge\left(\square p_{1} \wedge \square p_{2} \leftrightarrow \square\left(p_{1} \wedge p_{2}\right)\right)$, then

$$
T_{\psi}=\mathbf{f}(\mathbf{1}) \wedge\left(\mathbf{f}\left(\mathbf{x}_{1}\right) \wedge \mathbf{f}\left(\mathbf{x}_{2}\right) \leftrightarrow \mathbf{f}\left(\mathbf{x}_{1} \wedge \mathbf{x}_{2}\right)\right) .
$$

Lemma 2. Let $\varphi$ be a propositional formula with proposition symbols $p_{1}, \ldots, p_{r}$ and let $\mathcal{M}=\langle W, F, V\rangle$ be a Scott-Montague model. Let $\mathbf{a}_{j}=\mathbf{a}_{V\left(p_{j}\right)}$ for all $1 \leq j \leq r$. Then for all $1 \leq i \leq n$,

$$
\mathcal{M}, w_{i} \models \varphi \text { if and only if } G_{\varphi}\left(\mathbf{a}_{1}, \ldots, \mathbf{a}_{r}\right)[i]=1,
$$

i.e. $G_{\varphi}\left(\mathbf{a}_{1}, \ldots, \mathbf{a}_{r}\right)=\mathbf{a}_{\|\varphi\| \mathcal{M}}$.

Proof. The lemma is easily proved by induction on the construction of $\varphi$, similarly to the proof of Lemma 1.

Theorem 3. Let $\psi$ be a uniform degree-1 formula $\varphi\left(\square \varphi_{1}, \ldots, \square \varphi_{m}\right)$ and let $p_{1}, \ldots, p_{r}$ be the proposition symbols which occur in $\psi$. Let $\mathcal{M}=\langle W, F, V\rangle$ be a Scott-Montague model and let $\mathbf{a}_{j}=\mathbf{a}_{V\left(p_{j}\right)}$ for all $1 \leq j \leq r$. Then for all $1 \leq i \leq n$,

$$
\mathcal{M}, w_{i} \models \psi \text { if and only if } T_{\psi}\left(f_{F}, \mathbf{a}_{1}, \ldots, \mathbf{a}_{r}\right)[i]=1 \text {. }
$$

Proof. The proof is by induction on the construction of $\varphi$. The cases where $\varphi$ is a constant $\perp$ or $T$, or of the form $\neg \theta$ or $\theta_{1} \wedge \theta_{2}$, are straightforward. If $\varphi\left(p_{1}, \ldots, p_{m}\right)=p_{j}$ for some $1 \leq j \leq m$, then $\psi=\square \varphi_{j}$ and therefore $T_{\psi}=$ $\mathbf{f}\left(G_{\varphi_{j}}\left(\mathbf{x}_{1}, \ldots, \mathbf{x}_{r}\right)\right)$. Hence

$$
\begin{aligned}
\mathcal{M}, w_{i}=\psi & \Leftrightarrow \mathcal{M}, w_{i} \models \square \varphi_{j} \\
& \Leftrightarrow w_{i} \in F\left(\left\|\varphi_{j}\right\|^{\mathcal{M}}\right) \\
& \Leftrightarrow f_{F}\left(\mathbf{a}_{\left\|\varphi_{j}\right\| \mathcal{M}}\right)[i]=1 \\
& \stackrel{(*)}{\Leftrightarrow} f_{F}\left(G_{\varphi_{j}}\left(\mathbf{a}_{1}, \ldots, \mathbf{a}_{r}\right)\right)[i]=1 \\
& \Leftrightarrow T_{\psi}\left(f_{F}, \mathbf{a}_{1}, \ldots, \mathbf{a}_{r}\right)[i]=1,
\end{aligned}
$$

where the equivalence $(*)$ holds by Lemma 2 .

From Theorem 3 we get immediately the intended corollary: 
Corollary 3. Let $\psi$ be a uniform degree-1 formula. Let $\mathcal{F}=\langle W, F\rangle$ be a ScottMontague frame and let $V$ be a function $\Phi \rightarrow \mathcal{P}(W)$. Let $p_{1}, \ldots, p_{r}$ be the proposition symbols which occur in $\psi$ and let $\mathbf{a}_{j}=\mathbf{a}_{V\left(p_{j}\right)}$ for all $1 \leq j \leq r$. Then

$$
\langle\mathcal{F}, V\rangle \models \psi \text { if and only if } T_{\psi}\left(f_{F}, \mathbf{a}_{1}, \ldots, \mathbf{a}_{r}\right)=\mathbf{1},
$$

and futhermore

$$
\mathcal{F} \models \psi \text { if and only if } T_{\psi}\left(f_{F}\right) \equiv \mathbf{1} .
$$

The following lemma and theorem show that the translations $T \mapsto \psi_{T}$ and $\psi \mapsto T_{\psi}$ are inverses of each other. Thus there is a one-to-one correspondence between functional terms and uniform degree- 1 formulas.

Lemma 3. Let $G$ be a propositional term and let $\varphi$ be a modal formula of degree 0 . Then $G_{\psi_{G}}=G$ and $\psi_{G_{\varphi}}=\varphi$.

Proof. Both claims are proved by a straightforward induction.

Theorem 4. Let $T$ be a functional term

$$
G\left(\mathbf{f}\left(G_{1}\left(\mathbf{x}_{1}, \ldots, \mathbf{x}_{r}\right), \ldots, \mathbf{f}\left(G_{m}\left(\mathbf{x}_{1}, \ldots, \mathbf{x}_{r}\right)\right)\right)\right.
$$

and let $\psi$ be a uniform degree-1 formula

$$
\varphi\left(\square \varphi_{1}, \ldots, \square \varphi_{m}\right) .
$$

Then $T_{\psi_{T}}=T$ and $\psi_{T_{\psi}}=\psi$.

Proof. The first claim is proved by induction on the construction of $G$. The cases where $G$ is a constant, or of the form $\neg H$ or $H_{1} \wedge H_{2}$, are straightforward. If $G\left(\mathbf{x}_{1}, \ldots, \mathbf{x}_{m}\right)=\mathbf{x}_{j}$ for some $1 \leq j \leq m$, then $T=\mathbf{f}\left(G_{j}\left(\mathbf{x}_{1}, \ldots, \mathbf{x}_{r}\right)\right)$ and hence $\psi_{T}=\square \psi_{G_{j}}$. Therefore

$$
\begin{aligned}
T_{\psi_{T}} & =\mathbf{f}\left(G_{\psi_{G_{j}}}\left(\mathbf{x}_{1}, \ldots, \mathbf{x}_{r}\right)\right) \\
& \stackrel{(*)}{=} \mathbf{f}\left(G_{j}\left(\mathbf{x}_{1}, \ldots, \mathbf{x}_{r}\right)\right) \\
& =T
\end{aligned}
$$

where the equality $(*)$ holds by Lemma 3 .

The second claim of the theorem is proved similarly by induction on the construction of $\varphi$.

Our main result is that definability of Boolean functions by functional terms corresponds exactly to definability of Scott-Montague frames by uniform degree-1 formulas. With this purpose in mind, we define a class of Scott-Montague frames corresponding to a class of Boolean functions and a class of Boolean functions corresponding to a class of Scott-Montague frames. Let $K$ be a class of Boolean functions and let $\mathcal{C}$ be a class of Scott-Montague frames. We denote by $\mathcal{C}_{K}$ the class of Scott-Montague frames

$$
\left\{\mathcal{F}_{f} \mid f \text { is a vector-valued Boolean function such that } f \in K\right\},
$$

and $K_{\mathcal{C}}$ denotes the class of Boolean functions

$$
\left\{g \in \Omega \mid \mathrm{g} \text { is a component of } f_{\mathcal{F}} \text {, where } \mathcal{F} \in \mathcal{C}\right\} .
$$


Theorem 5. (a) Let $K$ be the class of Boolean functions defined by a set $\mathcal{T}$ of functional terms. Then the class $\mathcal{C}_{K}$ is defined by $\Psi_{\mathcal{T}}$, where $\Psi_{\mathcal{T}}=\left\{\psi_{T} \mid T \in \mathcal{T}\right\}$.

(b) Let $\mathcal{C}$ be the class of Scott-Montague frames defined by a set $\Psi$ of uniform degree-1 formulas. Then the class $K_{\mathcal{C}}$ is defined by $\mathcal{T}_{\Psi}$, where $\mathcal{T}_{\Psi}=\left\{T_{\psi} \mid \psi \in \Psi\right\}$.

Proof. For (a), let $\mathcal{F}=\langle W, F\rangle$. If $\mathcal{F} \in \mathcal{C}_{K}$ then $\mathcal{F}=\mathcal{F}_{f}$ for some vectorvalued Boolean function $f \in K$. Now $T(f) \equiv \mathbf{1}$ for all $T \in \mathcal{T}$, and it follows from Corollary 2 that $\mathcal{F} \models \psi_{T}$ for all $T \in \mathcal{T}$. Therefore $\mathcal{F} \models \Psi_{\mathcal{T}}$. Suppose that $\mathcal{F} \models \Psi_{\mathcal{T}}$. Then $\mathcal{F} \models \psi_{T}$ for all $T \in \mathcal{T}$ and we get from Corollary 3 that $T_{\psi_{T}}\left(f_{F}\right) \equiv \mathbf{1}$ for all $T \in \mathcal{T}$. Since $T_{\psi_{T}}=T$ by Theorem 4 , we have that $T\left(f_{F}\right) \equiv \mathbf{1}$ for all $T \in \mathcal{T}$. Therefore $f_{F} \in K$, and since $F=F_{f_{F}}$ we conclude that $\mathcal{F}=\mathcal{F}_{f_{F}} \in \mathcal{C}_{K}$.

For (b), suppose first that $g \in K_{\mathcal{C}}$. Now $g$ is a component of a vector-valued Boolean function $f_{\mathcal{F}}$ where $\mathcal{F} \in \mathcal{C}$. Since $\mathcal{F}=\psi$ for all $\psi \in \Psi$, it follows from Corollary 3 that $T_{\psi}\left(f_{\mathcal{F}}\right) \equiv \mathbf{1}$ for all $\psi \in \Psi$. Since $g$ is a component of $f_{\mathcal{F}}$, we get that $T_{\psi}(g) \equiv 1$ for all $\psi \in \Psi$. Suppose then that $T_{\psi}(g) \equiv 1$ for all $\psi \in \Psi$. Let $\psi \in \Psi$ and let $g^{\prime}$ be a vector-valued Boolean function all of which components are $g$. Then $T_{\psi}\left(g^{\prime}\right) \equiv \mathbf{1}$ and it follows from Corollary 2 that $\mathcal{F}_{g^{\prime}}=\psi_{T_{\psi}}$. By Theorem 4, we know that $\psi_{T_{\psi}}=\psi$, and therefore $\mathcal{F}_{g^{\prime}} \models \psi$. So $\mathcal{F}_{g^{\prime}} \models \Psi$ and hence $\mathcal{F}_{g^{\prime}} \in \mathcal{C}$. Since $g$ is a component of $g^{\prime}$ and $g^{\prime}=f_{\mathcal{F}_{g^{\prime}}}$, we conclude that $g \in K_{\mathcal{C}}$.

Theorem 6. (a) Let $K$ be a class of Boolean functions defined by a set $\mathcal{T}$ of functional terms. Then $K_{\mathcal{C}_{K}}=K$.

(b) Let $\mathcal{C}$ be a class of Scott-Montague frames defined by a set $\Psi$ of uniform degree-1 formulas. Then $\mathcal{C}_{K_{\mathcal{C}}}=\mathcal{C}$.

Proof. To prove (a), let $g$ be a Boolean function. Since the class $K$ is defined by $\mathcal{T}$, it follows from Theorem 5 (a) that the class $\mathcal{C}_{K}$ is defined by the set $\Psi_{\mathcal{T}}$. Thus

$$
\begin{aligned}
g \in K_{\mathcal{C}_{K}} & \stackrel{(1)}{\Leftrightarrow} g \models T_{\psi} \text { for all } \psi \in \Psi_{\mathcal{T}} \\
& \Leftrightarrow g \models T_{\psi_{T}} \text { for all } T \in \mathcal{T} \\
& \stackrel{2}{\Leftrightarrow} g \models T \text { for all } T \in \mathcal{T} \\
& \Leftrightarrow g \in K,
\end{aligned}
$$

where the equivalence (1) holds by Theorem 5 (b) and (2) by Theorem 4 . Hence $K_{\mathcal{C}_{K}}=K$.

The proof of (b) is similar.

To summarize, Theorems 5 and 6 together with Theorem 4 tell us that a class $\mathcal{C}$ of Scott-Montague frames is definable by uniform degree- 1 formulas if and only if the corresponding class $K_{\mathcal{C}}$ of Boolean functions is defined by functional terms. Moreover, the defining functional terms are simply the translations of the defining formulas. Similarly a class $K$ of Boolean functions is definable by functional terms if and only if the corresponding class $\mathcal{C}_{K}$ of Scott-Montague frames is definable by uniform degree- 1 formulas, and the defining formulas are the translations of the defining functional terms. 
Theorem 1 gave a characterization for definability of classes of Boolean functions by functional terms. Using Theorems 1 and 5, one can easily derive a similar characterization for definability of classes of Scott-Montague frames by uniform degree- 1 formulas. That is, a class $\mathcal{C}$ of Scott-Montague frames $\mathcal{F}$ is definable by a set of uniform degree- 1 formulas if and only if $\mathcal{C}$ satisfies the following closure condition:

If every component $f_{i}$ of $f_{\mathcal{F}}$ can be represented as $f_{i}=g\left(x_{j_{1}}, \ldots, x_{j_{n}}\right)$ where $g$ is a component of $f_{\mathcal{F}^{\prime}}$ for some $\mathcal{F}^{\prime} \in \mathcal{C}$, then $\mathcal{F} \in \mathcal{C}$.

This condition can be formulated in terms of the set function $F$, without using the component functions, but in that case the formulation is very technical and therefore more difficult to understand.

\section{Kripke correspondence}

In addition to the general correspondence, there are also connections between classes of Kripke frames and Boolean clones. This is not so surprising since Kripke frames are just special cases of Scott-Montague frames. In this section we show that several classes of Kripke frames correspond to classes of Scott-Montague frames which are defined by the translations $\psi_{T}$ of functional terms $T$ defining some Boolean clones.

Let $\mathcal{M}$ and $\mathcal{M}^{\prime}$ be Kripke or Scott-Montague models with a universe $W$ and a valuation $V$. We say that the models $\mathcal{M}$ and $\mathcal{M}^{\prime}$ are pointwise equivalent if for all $w \in W$ and for all formulas $\varphi$,

$$
\mathcal{M}, w \models \varphi \text { if and only if } \mathcal{M}^{\prime}, w \models \varphi .
$$

It is well known that there is a one-to-one correspondence between Kripke frames and so-called augmented Scott-Montague frames such that for all valuations the models based on these frames are pointwise equivalent. We will give this correspondence on finite frames in the next two lemmas and Proposition 2. In this section we consider only finite frames, in which case augmented Scott-Montague frames are just frames in which the axioms $\square \top$ and $\square p \wedge \square q \leftrightarrow \square(p \wedge q)$ are valid. If we would also consider infinite frames, then we would need an additional condition on Scott-Montague frames. For the correspondence and more information on augmented Scott-Montague frames, see [3].

Lemma 4. Let $\langle W, R\rangle$ be a Kripke frame and let $\left\langle W, F_{R}\right\rangle$ be a Scott-Montague frame where $F_{R}$ is given by

$$
F_{R}(X)=\{w \in W \mid \forall v \in W: w R v \Rightarrow v \in X\} \text { for all } X \subseteq W .
$$

Then for all valuations $V$, the models $\langle W, R, V\rangle$ and $\left\langle W, F_{R}, V\right\rangle$ are pointwise equivalent. Furthermore the axioms $\square \top$ and $\square p \wedge \square q \leftrightarrow \square(p \wedge q)$ are valid in the frame $\left\langle W, F_{R}\right\rangle$.

Let $\langle W, R\rangle$ be a Kripke frame and let $w \in W$. By $R[w]$ we denote the set of $R$-successors of $w$, i.e. $R[w]=\{v \in W \mid w R v\}$. 
Lemma 5. Let $\langle W, F\rangle$ be a Scott-Montague frame which validates the axioms $\square \top$ and $\square p \wedge \square q \leftrightarrow \square(p \wedge q)$ and let $f=f_{F}$. Let $\left\langle W, R_{F}\right\rangle$ be a Kripke frame where $R_{F}$ is given by

$$
R_{F}\left[w_{i}\right]=\left\{w_{j} \in W \mid x_{j} \text { is an essential variable of } f_{i}\right\} \text { for all } w_{i} \in W,
$$

where $f_{i}$ is the ith component function of $f$. Then for all valuations $V$, the models $\langle W, F, V\rangle$ and $\left\langle W, R_{F}, V\right\rangle$ are pointwise equivalent.

Proof. The clone $\Lambda_{1}$ is defined by the functional terms

$$
\mathbf{f}(\mathbf{1}), \mathbf{f}(\mathbf{x}) \wedge \mathbf{f}(\mathbf{y}) \leftrightarrow \mathbf{f}(\mathbf{x} \wedge \mathbf{y}),
$$

and so, by Theorem 5 , the class $\mathcal{C}_{\Lambda_{1}}$ of Scott-Montague frames is defined by the axioms $\square T$ and $(\square p \wedge \square q) \leftrightarrow \square(p \wedge q)$. Thus $\langle W, F\rangle \in \mathcal{C}_{\Lambda_{1}}$. Let $f_{i}$ be a component function of $f$. Then $f_{i} \in K_{\mathcal{C}_{\Lambda_{1}}}$ and it follows from Theorem 6 that $f_{i} \in \Lambda_{1}$. Hence, as observed in Remark 1,

$$
f_{i}(\mathbf{a})=1 \text { if and only if } \mathbf{a}[j]=1 \text { for every essential variable } x_{j} \text { of } f_{i} \text {. }
$$

Let $V$ be a valuation and let $\mathcal{M}=\langle W, F, V\rangle$ and $\mathcal{M}^{\prime}=\left\langle W, R_{F}, V\right\rangle$. We have to show that for every formula $\varphi$ and for every $w_{i} \in W$,

$$
\mathcal{M}, w_{i}=\varphi \text { if and only if } \mathcal{M}^{\prime}, w_{i}=\varphi .
$$

We proceed by induction on the construction of $\varphi$. Clearly, the claim holds for constants and proposition symbols. Using the induction hypothesis, it is easy to verify that the claim also holds for $\varphi=\neg \psi$ and $\varphi=\psi_{1} \wedge \psi_{2}$. Suppose that $\varphi=\square \psi$. By the induction hypothesis, we have that $\|\psi\|^{\mathcal{M}}=\|\psi\|^{\mathcal{M}^{\prime}}$ and therefore

$$
\begin{aligned}
\mathcal{M}, w_{i} \models \square \psi & \Leftrightarrow w_{i} \in F\left(\|\psi\|^{\mathcal{M}}\right) \\
& \Leftrightarrow f_{i}\left(\mathbf{a}_{\|\psi\| \mathcal{M}}\right)=1 \\
& \Leftrightarrow \mathbf{a}_{\|\psi\| \mathcal{M}}[j]=1 \text { for every essential variable } x_{j} \text { of } f_{i} \\
& \Leftrightarrow w_{j} \in\|\psi\|^{\mathcal{M}} \text { for every essential variable } x_{j} \text { of } f_{i} \\
& \Leftrightarrow w_{j} \in\|\psi\|^{\mathcal{M}^{\prime}} \text { for every } w_{j} \in R_{F}\left[w_{i}\right] \\
& \Leftrightarrow \mathcal{M}^{\prime}, w_{i}=\square \psi .
\end{aligned}
$$

By the induction principle it follows that the claim holds for every formula $\varphi$, which completes the proof of the lemma.

Lemma 6. Let $\langle W, R\rangle$ be a Kripke frame and let $F_{R}$ be defined as in Lemma 4. Let $f_{i}$ be the ith component of $f_{F_{R}}$. Then

$$
x_{j} \text { is an essential variable of } f_{i} \text { if and only if } w_{j} \in R\left[w_{i}\right] \text {. }
$$

Proof. By Lemma 4 axioms $\square \top$ and $\square p \wedge \square q \leftrightarrow \square(p \wedge q)$ are valid in $\left\langle W, F_{R}\right\rangle$. Thus $\left\langle W, F_{R}\right\rangle \in \mathcal{C}_{\Lambda_{1}}$ and hence $f_{i} \in \Lambda_{1}$ as shown in the proof of Lemma 5. Let $W=\left\{w_{1}, \ldots, w_{n}\right\}$. From the definition of the function $F_{R}$ we get that for every $1 \leq i \leq n$ and $\mathbf{a} \in \mathbb{B}^{n}$,

$$
f_{i}(\mathbf{a})=1 \Leftrightarrow\left(\forall k: w_{i} R w_{k} \Rightarrow \mathbf{a}[k]=1\right) .
$$

From this fact, one can see that if $w_{j} \notin R\left[w_{i}\right]$ then for all $\mathbf{a} \in \mathbb{B}^{n}$, the value of $f_{i}(\mathbf{a})$ does not depend on the $j$ th component of $\mathbf{a}$. Hence, if $w_{j} \notin R\left[w_{i}\right]$ then $x_{j}$ is not an essential variable of $f_{i}$. 
Suppose then that $w_{i} R w_{j}$. Since $f_{i} \in \Lambda_{1}$, we have that $f_{i}(\mathbf{1})=1$. Let $\mathbf{a} \in \mathbb{B}^{n}$ such that $\mathbf{a}[j]=0$ and $\mathbf{a}[k]=1$ for every $k \neq j$. Since $w_{i} R w_{j}$ and $\mathbf{a}[j]=0$ we get that $f_{i}(\mathbf{a})=0$. Thus $f_{i}(\mathbf{1}) \neq f_{i}(\mathbf{a})$ and so $x_{j}$ is an essential variable of $f_{i}$.

Proposition 2. Let $\langle W, R\rangle$ be a Kripke frame, let $\langle W, F\rangle \in \mathcal{C}_{\Lambda_{1}}$ and let $F_{R}$ and $R_{F}$ be defined as in Lemmas 4 and 5. Then the translations $R \mapsto F_{R}$ and $F \mapsto R_{F}$ are inverses of each other, i.e. $R_{F_{R}}=R$ and $F_{R_{F}}=F$.

Proof. We show first that $R_{F_{R}}=R$. Let $w_{i}, w_{j} \in W$ and let $f_{i}$ be the $i$ th component of $f_{F_{R}}$. Then

$$
\begin{aligned}
w_{j} \in R_{F_{R}}\left[w_{i}\right] & \Leftrightarrow x_{j} \text { is an essential variable of } f_{i} \\
& \Leftrightarrow w_{j} \in R\left[w_{i}\right],
\end{aligned}
$$

where the first equivalence holds by the definition of $R_{F_{R}}$ and the second equivalence holds by Lemma 6 . Therefore $R_{F_{R}}=R$.

To prove the second claim of the proposition, let $w_{i} \in W$ and $X \subseteq W$. Let $g=\left(g_{1}, \ldots, g_{n}\right)$ be the vector-valued Boolean function determined by $F_{R_{F}}$, and let $f_{i}$ be the $i$ th component of $f_{F}$. Since $\langle W, F\rangle \in \mathcal{C}_{\Lambda_{1}}$, we know that $f_{i} \in \Lambda_{1}$. Thus

$$
\begin{aligned}
w_{i} \in F_{R_{F}}(X) & \Leftrightarrow g_{i}\left(\mathbf{a}_{X}\right)=1 \\
& \stackrel{(1)}{\Leftrightarrow} \forall k: w_{i} R_{F} w_{k} \Rightarrow \mathbf{a}_{X}[k]=1 \\
& \stackrel{2}{\Leftrightarrow} \mathbf{a}_{X}[k]=1 \text { for every essential variable } x_{k} \text { of } f_{i} \\
& \stackrel{(3)}{\Leftrightarrow} f_{i}\left(\mathbf{a}_{X}\right)=1 \\
& \Leftrightarrow w_{i} \in F(X) .
\end{aligned}
$$

The equivalence (1) is explained in the proof of Lemma 6 , the equivalence (2) holds by the definition of $R_{F}$ and the equivalence (3) follows from Remark 1 . Hence, we conclude that $F_{R_{F}}=F$.

Let $\mathcal{K}$ be a class of Kripke frames and let $\mathcal{C}$ be a class of Scott-Montague frames. We say that the class $\mathcal{K}$ corresponds to the class $\mathcal{C}$ if there are translations $R \mapsto F_{R}$ and $F \mapsto R_{F}$ such that the following conditions hold.

- If $\langle W, R\rangle \in \mathcal{K}$, then $\left\langle W, F_{R}\right\rangle \in \mathcal{C}$, and for all valuations $V$, the models $\langle W, R, V\rangle$ and $\left\langle W, F_{R}, V\right\rangle$ are pointwise equivalent.

- If $\langle W, F\rangle \in \mathcal{C}$, then $\left\langle W, R_{F}\right\rangle \in \mathcal{K}$, and for all valuations $V$, the models $\langle W, F, V\rangle$ and $\left\langle W, R_{F}, V\right\rangle$ are pointwise equivalent.

- The translations $R \mapsto F_{R}$ and $F \mapsto R_{F}$ are inverses of each other.

Furthermore, we say that the class $\mathcal{K}$ corresponds to a Boolean clone $C$ if $\mathcal{K}$ corresponds to the class $\mathcal{C}_{C}$ of Scott-Montague frames. From Lemmas 4 and 5 and Proposition 2 we obtain the following proposition.

Proposition 3. Let $\mathcal{K}$ be the class of all Kripke frames. Then $\mathcal{K}$ corresponds to the clone $\Lambda_{1}$.

Remark 2. Let $\langle W, F\rangle$ be a Scott-Montague frame. Since the class $\mathcal{C}_{\Lambda_{1}}$ is defined by the axioms $\square T$ and $\square p \wedge \square q \leftrightarrow \square(p \wedge q)$, it is easy to see that $\langle W, F\rangle \in \mathcal{C}_{\Lambda_{1}}$ if and only if $F$ satisfies the conditions

$$
F(W)=W \text { and }
$$




$$
F(X) \cap F(Y)=F(X \cap Y)
$$

hold for all $X, Y \subseteq W$.

We also get interesting correspondence results between some subclasses of $\mathcal{K}$ and subclones of $\Lambda_{1}$. For example, there is a class of Kripke frames which corresponds to the minimal clone $I_{c}$. This clone plays a key role when characterizing definability of classes of Boolean functions by functional terms, see Theorem 1 and the discussion before it. For this correspondence result, we need to consider Kripke frames $\mathcal{F}=$ $\langle W, R\rangle$ where $R$ is a serial relation, i.e. every $w \in W$ has an $R$-successor. In the following propositions we use the same translations $R \mapsto F_{R}$ and $F \mapsto R_{F}$ as in Lemmas 4 and 5. While Lemmas 4 and 5 and Proposition 2 do the most of the work of the proofs, we need to show that the frames obtained from the translations belong to the right classes of Kripke and Scott-Montague frames in question.

Proposition 4. Let $\mathcal{K}_{s}$ be the class of all Kripke frames $\mathcal{F}=\langle W, R\rangle$ such that $R$ is a serial relation. Then $\mathcal{K}_{s}$ corresponds to the clone $\Lambda_{c}$.

Proof. The clone $\Lambda_{c}$ is defined by the functional terms

$$
\neg \mathbf{f}(\mathbf{0}), \mathbf{f}(\mathbf{1}), \mathbf{f}(\mathbf{x}) \wedge \mathbf{f}(\mathbf{y}) \leftrightarrow \mathbf{f}(\mathbf{x} \wedge \mathbf{y})
$$

and so the class $\mathcal{C}_{\Lambda_{c}}$ is defined by the axioms $\neg \square \perp, \square \top$ and $(\square p \wedge \square q) \leftrightarrow \square(p \wedge q)$. It is easy to verify that a Scott-Montague frame $\mathcal{F}=\langle W, F\rangle$ validates these axioms if and only if the conditions

(1) $F(\emptyset)=\emptyset$

(2) $F(W)=W$ and

(3) $F(X) \cap F(Y)=F(X \cap Y)$

hold for every $X, Y \subseteq W$.

Let $\langle W, R\rangle \in \mathcal{K}_{s}$. Now $R[w] \neq \emptyset$ for every $w \in W$ and it is immediate from the definition of $F_{R}$ that $F_{R}(\emptyset)=\emptyset$. It follows from Lemma 4 that $\left\langle W, F_{R}\right\rangle \in \mathcal{C}_{\Lambda_{1}}$ and thus, by Remark $2, F_{R}$ satisfies conditions (2) and (3). Hence $\left\langle W, F_{R}\right\rangle \in \mathcal{C}_{\Lambda_{c}}$.

Let $\langle W, F\rangle \in \mathcal{C}_{\Lambda_{c}}$. Now $\langle W, F\rangle \models \neg \square \perp$ and it follows from Lemma 5 that also $\left\langle W, R_{F}\right\rangle=\neg \square \perp$. From this fact, one can easily conclude that $R_{F}$ has to be a serial relation and hence $\left\langle W, R_{F}\right\rangle \in \mathcal{K}_{s}$.

Proposition 5. Let $\mathcal{K}_{f}$ be the class of all Kripke frames $\mathcal{F}=\langle W, R\rangle$ such that $R$ is a function. Then $\mathcal{K}_{f}$ corresponds to the clone $I_{c}$.

Proof. The clone $I_{c}$ is defined by the functional terms

$$
\mathbf{f}(\mathbf{1}), \mathbf{f}(\mathbf{x}) \wedge \mathbf{f}(\mathbf{y}) \leftrightarrow \mathbf{f}(\mathbf{x} \wedge \mathbf{y}), \neg \mathbf{f}(\mathbf{x}) \leftrightarrow \mathbf{f}(\neg \mathbf{x})
$$

Hence the class $\mathcal{C}_{I_{c}}$ is defined by the axioms $\square \top,(\square p \wedge \square q) \leftrightarrow \square(p \wedge q)$ and $\neg \square p \leftrightarrow \square \neg p$. It is easy to check that these axioms are valid in a Scott-Montague frame $\mathcal{F}=\langle W, F\rangle$ if and only if the conditions

(1) $F(W)=W$

(2) $F(X) \cap F(Y)=F(X \cap Y)$ and

(3) $\overline{F(X)}=F(\bar{X})$ 
hold for every $X, Y \subseteq W$.

Let $\langle W, R\rangle \in \mathcal{K}_{f}$. We show that $\overline{F_{R}(X)}=F_{R}(\bar{X})$ for all $X \subseteq W$. Let $w \in W$ and $X \subseteq W$. Since $\langle W, R\rangle \in \mathcal{K}_{f}$, the relation $R$ is a function and we have that

$$
\begin{aligned}
w \in \overline{F_{R}(X)} & \Leftrightarrow w \notin F_{R}(X) \\
& \Leftrightarrow \exists v \in W: w R v \wedge v \in \bar{X} \\
& \Leftrightarrow \forall v \in W: w R v \Rightarrow v \in \bar{X} \\
& \Leftrightarrow w \in F_{R}(\bar{X}) .
\end{aligned}
$$

Hence $F_{R}$ satisfies the condition (3). As before, $F_{R}$ satisfies also the conditions (1) and (2), and so $\left\langle W, F_{R}\right\rangle \in \mathcal{C}_{I_{c}}$.

Let $\langle W, F\rangle \in \mathcal{C}_{I_{c}}$. Since $\langle W, F\rangle \models \neg \square p \leftrightarrow \square \neg p$, it follows from Lemma 5 that $\left\langle W, R_{F}\right\rangle \models \neg \square p \leftrightarrow \square \neg p$. We have to show that $R_{F}$ is a function. Suppose on the contrary that $R_{F}$ is not a function. Then either some element of $W$ does not have an $R_{F}$-successor or some element of $W$ has at least two of them. Assume first that there is $w \in W$ which has no $R_{F}$-successor. Let $\mathcal{M}=\left\langle W, R_{F}, V\right\rangle$ be a Kripke model. Then trivially $\mathcal{M}, w \models \square \neg p$ and $\mathcal{M}, w \not \models \neg \square p$. Thus the axiom $\neg \square p \leftrightarrow \square \neg p$ is not valid in the frame $\left\langle W, R_{F}\right\rangle$. Assume then that there is $w \in W$ which has two $R_{F}$-successors $w_{1}$ and $w_{2}$. Consider a Kripke model $\mathcal{M}=\left\langle W, R_{F}, V\right\rangle$ where $V$ is a valuation such that $V(p)=\left\{w_{1}\right\}$. Then $\mathcal{M}, w \models \neg \square p$ and $\mathcal{M}, w \not \models \square \neg p$. Hence the axiom $\neg \square p \leftrightarrow \square \neg p$ is not valid in the frame $\left\langle W, R_{F}\right\rangle$. So, both cases lead up to a contradiction and therefore $R_{F}$ is a function.

Proposition 6. Let $\mathcal{K}_{p}$ be the class of all Kripke frames $\mathcal{F}=\langle W, R\rangle$ such that $R$ is a partial function. Then $\mathcal{K}_{p}$ corresponds to the clone $I_{1}$.

Proof. The clone $I_{1}$ is defined by the functional terms

$$
\mathbf{f}(\mathbf{1}), \mathbf{f}(\mathbf{x}) \wedge \mathbf{f}(\mathbf{y}) \leftrightarrow \mathbf{f}(\mathbf{x} \wedge \mathbf{y}), \neg \mathbf{f}(\mathbf{x}) \rightarrow \mathbf{f}(\neg \mathbf{x}) .
$$

Thus the class $\mathcal{C}_{I_{1}}$ of Scott-Montague frames is defined by the axioms $\square \top, \square p \wedge$ $\square q \leftrightarrow \square(p \wedge q)$ and $\neg \square p \rightarrow \square \neg p$. These axioms are valid in a Scott-Montague frame $\mathcal{F}=\langle W, F\rangle$ if and only if the conditions

(1) $F(W)=W$,

(2) $F(X) \cap F(Y)=F(X \cap Y)$ and

(3) $\overline{F(X)} \subseteq F(\bar{X})$

hold for every $X, Y \subseteq W$.

Let $\langle W, F\rangle \in \mathcal{K}_{p}$. Again $F_{R}$ satisfies the conditions (1) and (2). We will show that $\overline{F_{R}(X)} \subseteq F_{R}(\bar{X})$. Let $X \subseteq W$ and let $w \in \overline{F_{R}(X)}$. Then $w \notin F_{R}(X)$ and thus there exists $v \in W$ such that $w R v$ and $v \notin X$. Since $R$ is a partial function, there is only one $v$ such that $w R v$. Hence we have that for all $v \in W, w R v$ implies $v \in \bar{X}$, and therefore $w \in F_{R}(\bar{X})$. Thus $\overline{F_{R}(X)} \subseteq F_{R}(\bar{X})$ and we have proved that $\left\langle W, F_{R}\right\rangle \in \mathcal{C}_{I_{1}}$.

Let then $\langle W, F\rangle \in \mathcal{C}_{I_{1}}$. We know that $\langle W, F\rangle \models \neg \square p \rightarrow \square \neg p$ and hence, by Lemma $5,\left\langle W, R_{F}\right\rangle \models \neg \square p \rightarrow \square \neg p$. We have to show that $\left\langle W, R_{F}\right\rangle \in \mathcal{K}_{p}$, i.e. $R_{F}$ is a partial function. If $R_{F}$ is not a partial function then there is $w \in W$ which has two $R_{F}$-successors. By proceeding as in the proof of Proposition 5 , one can 
show that the axiom $\neg \square p \rightarrow \square \neg p$ is not valid in the frame $\left\langle W, R_{F}\right\rangle$. This is a contradiction and thus $R_{F}$ has to be a partial function.

Note that if we had chosen $\diamond$ as the basic operator in modal logic and given the truth condition of $\diamond$ in Scott-Montague semantics by the set function, then the class of all Kripke frames would correspond to the clone $V_{0}$, which is the dual of $\Lambda_{1}$. Similarly, the classes $\mathcal{K}_{s}$ and $\mathcal{K}_{p}$ would correspond to the duals of $\Lambda_{c}$ and $I_{1}$, respectively, i.e. to the clones $V_{c}$ and $I_{0}$. Since the clone $I_{c}$ is self-dual, the class $\mathcal{K}_{f}$ would still correspond to $I_{c}$.

\section{Linear classes}

In this section we establish further correspondences between classes of Kripke frames and clones of Boolean functions by modifying the standard Kripke semantics. These modifications are based on the parity of the sets of $R$-successors. As in the previous section, we consider only finite frames.

Throughout this section we make use of the exclusive-or, denoted by $\oplus$ and defined by

$$
\varphi \oplus \psi=(\varphi \wedge \neg \psi) \vee(\neg \varphi \wedge \psi)
$$

as a propositional connective in formulas of modal logic. One can easily verify that $\oplus$ is associative and commutative. Furthermore, for every Kripke or ScottMontague model $\mathcal{M}$ with universe $W$ and for every $w \in W, \mathcal{M}, w \models \varphi_{1} \oplus \cdots \oplus \varphi_{m}$ if and only if $\mathcal{M}, w \models \varphi_{i}$ for an odd number of indices $1 \leq i \leq m$.

Let $X, Y \subseteq W$. By $\bar{X}$ we denote the complement of $X$ with respect to $W$. We use the symbol $\oplus$ also for the symmetric difference

$$
X \oplus Y=(X \backslash Y) \cup(Y \backslash X)=(X \cap \bar{Y}) \cup(Y \cap \bar{X}) .
$$

Similarly to the exclusive-or, the symmetric difference is associative and commutative.

The frames and models that we shall consider are essentially the same as those used in Kripke semantics. In fact, the semantics that we will introduce differ from Kripke semantics only in the account for the modal operator $\square$. However, the notion of correspondence between classes of Kripke and Scott-Montague frames must be also modified accordingly. In the sequel we will define three new truth relations, $\models_{O}, \models_{S}$ and $\models_{M}$, which give the modifications of Kripke semantics that we are going to consider.

Let $\mathcal{M}=\langle W, R, V\rangle$ be a Kripke model and let $\mathcal{M}^{\prime}=\langle W, F, V\rangle$ be a ScottMontague model. We say that the models $\mathcal{M}$ and $\mathcal{M}^{\prime}$ are pointwise equivalent with respect to $\models_{\eta}$, where $\eta \in\{O, S, M\}$, if for all modal formulas $\varphi$ and for all $w \in W$,

$$
\mathcal{M}, w \models_{\eta} \varphi \text { if and only if } \mathcal{M}^{\prime}, w \models \varphi \text {. }
$$

Let $\left(\mathcal{K}, \models_{\eta}\right)$ be a pair where $\mathcal{K}$ is a class of Kripke frames and $\models_{\eta}$ is the truth relation referring to the semantics which is being used, and let $\mathcal{C}$ be a class of Scott-Montague frames. We say that the pair $\left(\mathcal{K}, \models_{\eta}\right)$ corresponds to the class $\mathcal{C}$ if there are translations $R \mapsto F_{R}$ and $F \mapsto R_{F}$ such that the following conditions hold. 
- If $\langle W, R\rangle \in \mathcal{K}$, then $\left\langle W, F_{R}\right\rangle \in \mathcal{C}$, and for all valuations $V$, the models $\langle W, R, V\rangle$ and $\left\langle W, F_{R}, V\right\rangle$ are pointwise equivalent with respect to $=_{\eta}$.

- If $\langle W, F\rangle \in \mathcal{C}$, then $\left\langle W, R_{F}\right\rangle \in \mathcal{K}$, and for all valuations $V$, the models $\left\langle W, R_{F}, V\right\rangle$ and $\langle W, F, V\rangle$ are pointwise equivalent with respect to $\models_{\eta}$.

- The translations $R \mapsto F_{R}$ and $F \mapsto R_{F}$ are inverses of each other.

As in the previous section, we say that the pair $\left(\mathcal{K}, \models_{\eta}\right)$ corresponds to a clone $C$ if the pair $\left(\mathcal{K}, \models_{\eta}\right)$ corresponds to the class $\mathcal{C}_{C}$.

We will provide pairs $\left(\mathcal{K}, \models_{\eta}\right)$ for each of the linear clones $L, L_{0}, L_{1}, L_{c}$ and $L S$. Consider first the clone $L_{0}$ which is defined by the functional term

$$
\mathbf{1} \oplus \mathbf{f}(\mathbf{x}) \oplus \mathbf{f}(\mathbf{y}) \oplus \mathbf{f}(\mathbf{x} \oplus \mathbf{y}) .
$$

It follows from Theorem 5 that the class $\mathcal{C}_{L_{0}}$ is defined by the axiom $\top \oplus \square p \oplus \square q \oplus$ $\square(p \oplus q)$. It is easy to verify that this axiom is valid in a Scott-Montague frame $\mathcal{F}=\langle W, F\rangle$ if and only if the condition

(1) $F(X) \oplus F(Y)=F(X \oplus Y)$

holds for all $X, Y \subseteq W$.

The following lemma gives rise to one modification of Kripke semantics which will, together with the class of all Kripke frames, correspond to the clone $L_{0}$.

Lemma 7. Let $F: \mathcal{P}(W) \rightarrow \mathcal{P}(W)$ be a set function satisfying the condition (1). Let $R_{F}$ be the relation defined by

$$
R_{F}[w]=\{v \in W \mid w \in F(\{v\})\}
$$

for every $w \in W$. Then for all $X \subseteq W$ and $w \in W$,

$$
w \in F(X) \text { if and only if }\left|R_{F}[w] \cap X\right| \text { is odd. }
$$

Proof. Let $X \subseteq W$. First, note that if $X=X_{1} \oplus \cdots \oplus X_{k}$, then $w \in X$ if and only if $w \in X_{i}$ for an odd number of indices $i$. Since $F$ satisfies the condition (1) and $\oplus$ is associative, it is easy to see that $F\left(X_{1} \oplus \cdots \oplus X_{k}\right)=F\left(X_{1}\right) \oplus \cdots \oplus F\left(X_{k}\right)$. From the condition (1) we also get that $F(\emptyset)=F(\emptyset \oplus \emptyset)=F(\emptyset) \oplus F(\emptyset)=\emptyset$.

Let $w \in W$. Let $X^{\prime}$ denote the set $R_{F}[w] \cap X$, and suppose that $\left|X^{\prime}\right|$ is even. We show first that $w \notin F\left(X^{\prime}\right)$. If $X^{\prime}=\emptyset$ then $w \notin F\left(X^{\prime}\right)$, since $F(\emptyset)=\emptyset$. Let $X^{\prime}=\left\{w_{1}, \ldots, w_{2 m}\right\}$ for some $m \geq 1$. In other words, $X^{\prime}=\left\{w_{1}\right\} \oplus \cdots \oplus\left\{w_{2 m}\right\}$ and it follows from the definitions of $R_{F}[w]$ and $X^{\prime}$ that $w \in F\left(\left\{w_{i}\right\}\right)$ for all $1 \leq i \leq 2 m$. From this fact and the observations above, we get that

$$
w \notin F\left(\left\{w_{1}\right\}\right) \oplus \cdots \oplus F\left(\left\{w_{2 m}\right\}\right)=F\left(\left\{w_{1}\right\} \oplus \cdots \oplus\left\{w_{2 m}\right\}\right)=F\left(X^{\prime}\right) .
$$

Now we show that $w \notin F\left(X \backslash X^{\prime}\right)$. The case $\left(X \backslash X^{\prime}\right)=\emptyset$ is clear. Suppose that $\left(X \backslash X^{\prime}\right)=\left\{w_{1}, \ldots, w_{k}\right\}$ for some $k \geq 1$. By the definitions of $R_{F}[w]$ and $X^{\prime}$ we know that $w \notin F\left(\left\{w_{i}\right\}\right)$ for all $1 \leq i \leq k$. Thus

$$
w \notin F\left(\left\{w_{1}\right\}\right) \oplus \cdots \oplus F\left(\left\{w_{k}\right\}\right)=F\left(\left\{w_{1}\right\} \oplus \cdots \oplus\left\{w_{k}\right\}\right)=F\left(X \backslash X^{\prime}\right) .
$$

So $w \notin F\left(X^{\prime}\right)$ and $w \notin F\left(X \backslash X^{\prime}\right)$, and hence $w \notin F\left(X^{\prime}\right) \oplus F\left(X \backslash X^{\prime}\right)$. Since $X=X^{\prime} \oplus\left(X \backslash X^{\prime}\right)$, it follows from the condition (1) that

$$
w \notin F\left(X^{\prime}\right) \oplus F\left(X \backslash X^{\prime}\right)=F\left(X^{\prime} \oplus\left(X \backslash X^{\prime}\right)\right)=F(X) .
$$


Suppose now that $\left|X^{\prime}\right|$ is odd. Let $X^{\prime}=\left\{w_{1}, \ldots, w_{2 m-1}\right\}$ for some $m \geq 1$. Then $X^{\prime}=\left\{w_{1}\right\} \oplus \cdots \oplus\left\{w_{2 m-1}\right\}$ and since $w \in F\left(\left\{w_{i}\right\}\right)$ for all $1 \leq i \leq 2 m-1$, we have that

$$
w \in F\left(\left\{w_{1}\right\}\right) \oplus \cdots \oplus F\left(\left\{w_{2 m-1}\right\}\right)=F\left(\left\{w_{1}\right\} \oplus \cdots \oplus\left\{w_{2 m-1}\right\}\right)=F\left(X^{\prime}\right) .
$$

Furthermore $w \notin F\left(X \backslash X^{\prime}\right)$ and thus

$$
w \in F\left(X^{\prime}\right) \oplus F\left(X \backslash X^{\prime}\right)=F\left(X^{\prime} \oplus\left(X \backslash X^{\prime}\right)\right)=F(X) .
$$

Hence, we have proved the lemma.

The previous lemma motivates the following semantics. Let $\mathcal{M}=\langle W, R, V\rangle$ be a Kripke model and let $w \in W$. We define the truth relation $\models_{O}$ inductively for modal formulas. For constants, proposition symbols and Boolean connectives $\models_{O}$ is defined as $\models$ in Kripke semantics. Let $\varphi$ be a modal formula. For the modal operator $\square$, the truth relation is defined by

$$
\mathcal{M}, w=_{O} \square \varphi \text { iff }\left|R[w] \cap\|\varphi\|_{O}^{\mathcal{M}}\right| \text { is odd }
$$

where $\|\varphi\|_{O}^{\mathcal{M}}$ denotes the truth set of $\varphi$ in the model $\mathcal{M}$ with respect to $\models_{O}$.

We denote by $\mathcal{L}_{0}$ the pair $\left(\mathcal{K}, \models_{O}\right)$ where $\mathcal{K}$ is the class of all Kripke frames. We denote by $\mathcal{L}_{c}$ the pair $\left(\mathcal{K}_{O}, \models O\right)$ where $\mathcal{K}_{O}$ denotes the class of all Kripke frames $\langle W, R\rangle$ such that every $w \in W$ has an odd number of $R$-successors.

Proposition 7. The pair $\mathcal{L}_{0}$ corresponds to the clone $L_{0}$.

Proof. As mentioned before Lemma 7 , the class $\mathcal{C}_{L_{0}}$ consists of exactly those Scott-Montague frames $\langle W, F\rangle$ for which

(1) $F(X) \oplus F(Y)=F(X \oplus Y)$

holds for all $X, Y \subseteq W$.

The translation $R \mapsto F_{R}$ is given by

$$
F_{R}(X)=\{w \in W|| R[w] \cap X \mid \text { is odd }\}
$$

for all $X \subseteq W$ and the translation $F \mapsto R_{F}$ is defined as in Lemma 7 .

Let $\langle W, R\rangle \in \mathcal{K}$. We show that $\left\langle W, F_{R}\right\rangle \in \mathcal{C}_{L_{0}}$, i.e. $F_{R}$ satisfies the condition (1). Let $X, Y \subseteq W$ and let $w \in W$. We have to show that $w \in F_{R}(X) \oplus F_{R}(Y)$ if and only if $w \in F_{R}(X \oplus Y)$. It is obvious that the parities of the sets $R[w] \cap X$, $R[w] \cap Y$ and $R[w] \cap(X \oplus Y)$ depend only on the parities of the sets $R[w] \cap(X \backslash Y)$, $R[w] \cap(Y \backslash X)$ and $R[w] \cap(X \cap Y)$. So there are eight different cases. We consider here one of them, the other cases follow similarly. So suppose that $|R[w] \cap(X \backslash Y)|$ and $|R[w] \cap(Y \backslash X)|$ are odd and $|R[w] \cap(X \cap Y)|$ is even. Then $|R[w] \cap X|$ and $|R[w] \cap Y|$ are odd and $|R[w] \cap(X \oplus Y)|$ is even. So $w \in F_{R}(X), w \in F_{R}(Y)$ and $w \notin F_{R}(X \oplus Y)$. Thus $w \notin F_{R}(X) \oplus F_{R}(Y)$ and $w \notin F_{R}(X \oplus Y)$, and hence $w \in F_{R}(X) \oplus F_{R}(Y)$ if and only if $w \in F_{R}(X \oplus Y)$. The rest of the cases can be proved similarly, and therefore $\left\langle W, F_{R}\right\rangle \in \mathcal{C}_{L_{0}}$.

Let $V$ be a valuation and let $\mathcal{M}=\langle W, R, V\rangle$ and $\mathcal{M}^{\prime}=\left\langle W, F_{R}, V\right\rangle$. We show that the claim

$$
\mathcal{M}, w=_{O} \varphi \text { if and only if } \mathcal{M}^{\prime}, w \models \varphi
$$


holds for every modal formula $\varphi$ and $w \in W$. This is done by induction on the construction of $\varphi$. The base cases, $\varphi=\perp, \varphi=\top$ or $\varphi=p$, follow immediately from the definition of $\models_{O}$, and the cases, where $\varphi=\neg \psi$ or $\varphi=\psi_{1} \wedge \psi_{2}$, can be easily verified by making use of the induction hypothesis. So let $\varphi=\square \psi$ and suppose that $\psi$ satisfies the claim. Then $\|\psi\|_{O}^{\mathcal{M}}=\|\psi\|^{\mathcal{M}^{\prime}}$ and

$$
\begin{aligned}
\mathcal{M}, w \models_{O} \square \psi & \Leftrightarrow\left|R[w] \cap\|\psi\|_{O}^{\mathcal{M}}\right| \text { is odd } \\
& \Leftrightarrow\left|R[w] \cap\|\psi\|^{\mathcal{M}^{\prime}}\right| \text { is odd } \\
& \Leftrightarrow w \in F_{R}\left(\|\psi\|^{\mathcal{M}^{\prime}}\right) \\
& \Leftrightarrow \mathcal{M}^{\prime}, w \models \square \psi .
\end{aligned}
$$

Thus the models $\mathcal{M}$ and $\mathcal{M}^{\prime}$ are pointwise equivalent with respect to $\models_{O}$.

Let then $\langle W, F\rangle \in \mathcal{C}_{L_{0}}$. Since $R_{F}$ is a binary relation on $W$, we have that $\left\langle W, R_{F}\right\rangle \in \mathcal{K}$.

Let $V$ be a valuation and let $\mathcal{M}=\langle W, F, V\rangle$ and $\mathcal{M}^{\prime}=\left\langle W, R_{F}, V\right\rangle$. We show that for all formulas $\varphi$ and for all $w \in W$,

$$
\mathcal{M}, w \models \varphi \text { if and only if } \mathcal{M}^{\prime}, w \models_{O} \varphi \text {. }
$$

This is again done by induction. We consider only the case $\varphi=\square \psi$, the other cases are straightforward. So let $\varphi=\square \psi$ where $\psi$ satisfies the claim above. Then $\|\psi\|^{\mathcal{M}}=\|\psi\|_{O}^{\mathcal{M}^{\prime}}$ and

$$
\begin{aligned}
\mathcal{M}, w=\square \psi & \Leftrightarrow w \in F\left(\|\psi\|^{\mathcal{M}}\right) \\
& \stackrel{(*)}{\Leftrightarrow}\left|R_{F}[w] \cap\|\psi\|^{\mathcal{M}}\right| \text { is odd } \\
& \Leftrightarrow\left|R_{F}[w] \cap\|\psi\|_{O}^{\mathcal{M}^{\prime}}\right| \text { is odd } \\
& \Leftrightarrow \mathcal{M}^{\prime}, w \models_{O} \square \psi,
\end{aligned}
$$

where the equivalence $(*)$ holds by Lemma 7 . Hence the models $\mathcal{M}^{\prime}$ and $\mathcal{M}$ are pointwise equivalent with respect to $\models_{O}$.

Let $\langle W, R\rangle \in \mathcal{K}$ and let $\langle W, F\rangle \in \mathcal{C}_{L_{0}}$. We still have to prove that $R_{F_{R}}=R$ and $F_{R_{F}}=F$. Let $w \in W$ and $X \subseteq W$. Since for all $v \in W$,

$$
\begin{aligned}
v \in R_{F_{R}}[w] & \Leftrightarrow w \in F_{R}(\{v\}) \\
& \Leftrightarrow|R[w] \cap\{v\}| \text { is odd } \\
& \Leftrightarrow v \in R[w],
\end{aligned}
$$

and

$$
\begin{aligned}
w \in F_{R_{F}}(X) & \stackrel{\Leftrightarrow}{\Leftrightarrow}\left|R_{F}[w] \cap X\right| \text { is odd } \\
& \stackrel{(*)}{\Leftrightarrow} w \in F(X),
\end{aligned}
$$

where the equivalence $(*)$ holds again by Lemma 7 , we can conclude that $R_{F_{R}}=R$ and $F_{R_{F}}=F$.

Proposition 8. The pair $\mathcal{L}_{c}$ corresponds to the clone $L_{c}$.

Proof. The proof of Proposition 8 follows exactly the same steps as in the proof of Proposition 7 . The clone $L_{c}$ is defined by the functional terms

$$
\mathbf{f}(\mathbf{1}), \mathbf{1} \oplus \mathbf{f}(\mathbf{x}) \oplus \mathbf{f}(\mathbf{y}) \oplus \mathbf{f}(\mathbf{x} \oplus \mathbf{y}),
$$


and so the class $\mathcal{C}_{L_{c}}$ is defined by the axioms $\square \top$ and $\top \oplus \square p \oplus \square q \oplus \square(p \oplus q)$. These axioms are valid in a Scott-Montague frame $\mathcal{F}=\langle W, F\rangle$ if and only if the conditions

(i) $F(W)=W$ and

(ii) $F(X) \oplus F(Y)=F(X \oplus Y)$

hold for all $X, Y \subseteq W$.

The translations $R \mapsto F_{R}$ and $F \mapsto R_{F}$ are the same as in Proposition 7 .

Let $\langle W, R\rangle \in \mathcal{K}_{O}$. We show that $\left\langle W, F_{R}\right\rangle \in \mathcal{C}_{L_{c}}$. Since $|R[w]|$ is odd for every $w \in W$ it is clear that $F_{R}(W)=W$. By Lemma 7 , the function $F_{R}$ also satisfies the condition (ii). Thus $\left\langle W, F_{R}\right\rangle \in \mathcal{C}_{L_{c}}$.

Let $\langle W, F\rangle \in \mathcal{C}_{L_{c}}$. We have to show that $\left|R_{F}[w]\right|$ is odd for every $w \in W$. Let $w \in W$. Since $F(W)=W$, it follows from Lemma 7 that $R_{F}[w] \cap W$ has an odd number of elements. Since $R_{F}[w] \cap W=R_{F}[w]$, we have that $\left|R_{F}[w]\right|$ is odd, and therefore $\left\langle W, R_{F}\right\rangle \in \mathcal{K}_{O}$.

The remainder of the proof is similar to the proof of Proposition 7 .

Let $F$ be a set function $\mathcal{P}(W) \rightarrow \mathcal{P}(W)$. For the rest of the section we define the translation $F \mapsto R_{F}$ by

$$
R_{F}[w]=\{v \in W \mid w \in F(\{v\}) \Leftrightarrow w \notin F(\emptyset)\}
$$

for all $w \in W$. Note that this definition coincides with that in Lemma 7 because in that case $F(\emptyset)=\emptyset$.

The following lemma will be useful in the sequel.

Lemma 8. Let $F$ be a set function $\mathcal{P}(W) \rightarrow \mathcal{P}(W)$ which satisfies the condition

(2) $F(\emptyset) \oplus F(X) \oplus F(Y)=F(X \oplus Y)$

for all $X, Y \subseteq W$. Let $X \subseteq W$ and let $w \in W$.

(a) If $w \notin F(\emptyset)$, then $w \in F(X)$ if and only if $\left|R_{F}[w] \cap X\right|$ is odd.

(b) If $w \in F(\emptyset)$, then $w \in F(X)$ if and only if $\left|R_{F}[w] \cap X\right|$ is even.

Proof. As mentioned before, if $X=X_{1} \oplus \cdots \oplus X_{k}$ then $w \in X$ if and only if $w \in X_{i}$ for odd number of indices $i$. If $w \notin F(\emptyset)$ then $R_{F}[w]=\{v \in W \mid w \in$ $F(\{v\})\}$. We denote by $X^{\prime}$ the set $X \cap\{v \in W \mid w \in F(\{v\})\}$. And if $w \in F(\emptyset)$ then $R_{F}[w]=\{v \in W \mid w \notin F(\{v\})\}$. For the set $X \cap\{v \in W \mid w \notin F(\{v\})\}$ we use a notation $X^{\prime \prime}$.

To prove (a), suppose that $w \notin F(\emptyset)$. It follows from the condition (2) that

$$
w \in F(X) \oplus F(Y) \text { if and only if } w \in F(X \oplus Y)
$$

and from this, it is easy to see that $w \in F\left(X_{1} \oplus \cdots \oplus X_{k}\right)$ if and only if $w \in$ $F\left(X_{1}\right) \oplus \cdots \oplus F\left(X_{k}\right)$. Now the rest of the proof of (a) is essentially the same as the proof of Lemma 7 .

For the part (b), suppose that $w \in F(\emptyset)$. By using the condition (2), it is easy to see that $w \in F\left(X^{\prime}\right)$. In addition, an easy induction on the cardinality of $X^{\prime \prime}$ shows that $w \in F\left(X^{\prime \prime}\right)$ if and only if $\left|X^{\prime \prime}\right|$ is even. Since $w \in F(\emptyset)$ and $w \in F\left(X^{\prime}\right)$, we have that $w \in F(\emptyset) \oplus F\left(X^{\prime}\right) \oplus F\left(X^{\prime \prime}\right)$ if and only if $w \in F\left(X^{\prime \prime}\right)$. Since $X^{\prime} \oplus X^{\prime \prime}=X$, 
we get by the condition (2) that

$$
\begin{aligned}
w \in F(X) & \Leftrightarrow w \in F\left(X^{\prime} \oplus X^{\prime \prime}\right) \\
& \Leftrightarrow w \in F(\emptyset) \oplus F\left(X^{\prime}\right) \oplus F\left(X^{\prime \prime}\right) \\
& \Leftrightarrow w \in F\left(X^{\prime \prime}\right) \\
& \Leftrightarrow\left|X^{\prime \prime}\right| \text { is even. }
\end{aligned}
$$

This proves $(\mathrm{b})$.

Let $\mathcal{M}=\langle W, R, V\rangle$ be a Kripke model and let $w \in W$. The truth relation $\models_{S}$ is defined as $\models_{O}$ except for the modal operator $\square$, for which we define

$$
\mathcal{M}, w \models_{S} \square \varphi \text { iff the sets } R[w] \text { and } R[w] \cap\|\varphi\|_{S}^{\mathcal{M}} \text { have the same parity, }
$$

where $\|\varphi\|_{S}^{\mathcal{M}}$ denotes the truth set of $\varphi$ in the model $\mathcal{M}$ with respect to $\models_{S}$.

We denote by $\mathcal{L}_{1}$ the pair $\left(\mathcal{K}, \models_{S}\right)$ where $\mathcal{K}$ is the class of all Kripke frames.

Proposition 9. The pair $\mathcal{L}_{1}$ corresponds to the clone $L_{1}$.

Proof. The clone $L_{1}$ is defined by the functional terms

$$
\mathbf{f}(\mathbf{1}), \mathbf{1} \oplus \mathbf{f}(\mathbf{0}) \oplus \mathbf{f}(\mathbf{x}) \oplus \mathbf{f}(\mathbf{y}) \oplus \mathbf{f}(\mathbf{x} \oplus \mathbf{y}) .
$$

Thus the class $\mathcal{C}_{L_{1}}$ is defined by the axioms $\square \top$ and $\top \oplus \square \perp \oplus \square p \oplus \square q \oplus \square(p \oplus q)$. It is easy to verify that these axioms are valid in a Scott-Montague frame $\mathcal{F}=\langle W, F\rangle$ if and only if the conditions

(i) $F(W)=W$ and

(ii) $F(\emptyset) \oplus F(X) \oplus F(Y)=F(X \oplus Y)$

hold for all $X, Y \subseteq W$.

The translation $R \mapsto F_{R}$ is given by

$$
F_{R}(X)=\{w \in W|| R[w] \cap X \mid \text { is odd } \Leftrightarrow|R[w]| \text { is odd }\}
$$

for all $X \subseteq W$.

Let $\langle W, R\rangle \in \mathcal{K}$. We have to show that $\left\langle W, F_{R}\right\rangle \in \mathcal{C}_{L_{1}}$, i.e. $F_{R}$ satisfies the conditions (i) and (ii). It is immediate from the definition of $F_{R}$ that $F_{R}(W)=W$. It also follows straightforwardly from the definition of $F_{R}$ that for all $w \in W$, $w \in F_{R}(\emptyset)$ if and only if $|R[w]|$ is even. Using this fact one can prove, similarly as in the proof of Proposition 7, that for all $X, Y \subseteq W$ and for all $w \in W$,

$$
w \in F_{R}(\emptyset) \oplus F_{R}(X) \oplus F_{R}(Y) \text { if and only if } w \in F_{R}(X \oplus Y) \text {. }
$$

Thus $F_{R}$ satisfies also the condition (ii) and therefore $\left\langle W, F_{R}\right\rangle \in \mathcal{C}_{L_{1}}$.

Let $V$ be a valuation function and let $\mathcal{M}=\langle W, R, V\rangle$ and $\mathcal{M}^{\prime}=\left\langle W, F_{R}, V\right\rangle$. Similarly, as in the proof of Proposition 7, it can be proved that the models $\mathcal{M}$ and $\mathcal{M}^{\prime}$ are pointwise equivalent with respect to $\models_{S}$.

Let $\langle W, F\rangle \in \mathcal{C}_{L_{1}}$. Then $R_{F}$ is a relation on $W$ and $\left\langle W, R_{F}\right\rangle \in \mathcal{K}$.

Let $V$ be a valuation and let $\mathcal{M}=\langle W, F, V\rangle$ and $\mathcal{M}^{\prime}=\left\langle W, R_{F}, V\right\rangle$. By using Lemma 8, one can easily show that the models $\mathcal{M}^{\prime}$ and $\mathcal{M}$ are pointwise equivalent with respect to $\models S$.

Let $\langle W, R\rangle \in \mathcal{K}$ and $\langle W, F\rangle \in \mathcal{C}_{L_{1}}$. Our final task is to prove that $R_{F_{R}}=R$ and $F_{R_{F}}=F$. Let $w, v \in W$. Suppose that $v \in R_{F_{R}}[w]$ and $w \in F_{R}(\emptyset)$. Now it 
follows from the definition of $R_{F_{R}}$ that $w \notin F_{R}(\{v\})$. From the definition of $F_{R}$ we get that $|R[w] \cap\{v\}|$ is odd if and only if $|R[w]|$ is even. Since $w \in F_{R}(\emptyset)$ and $|R[w] \cap \emptyset|$ is even, it has to be the case that $|R[w]|$ is even. Thus $|R[w] \cap\{v\}|$ is odd and hence $v \in R[w]$. A similar proof shows that $v \in R[w]$ if $w \notin F_{R}(\emptyset)$.

Now suppose that $v \in R[w]$, i.e. $|R[w] \cap\{v\}|$ is odd. If $|R[w]|$ is also odd then $w \in F_{R}(\{v\})$ and $w \notin F_{R}(\emptyset)$ and thus $v \in R_{F_{R}}[w]$ by the definition of $R_{F_{R}}$. Also, if $|R[w]|$ is even, it is easy to see that $v \in R_{F_{R}}[w]$. Hence we have proved that $R_{F_{R}}=R$.

Similarly we can show that $F_{R_{F}}=F$.

For the remaining two linear clones, $L$ and $L S$, we consider Kripke frames with an additional unary relation $U \subseteq W$. Let $\mathcal{K}^{U}$ be the class of all frames $\mathcal{F}=\langle W, R, U\rangle$ where $U \subseteq W$. For the models based on these frames, we define a truth relation $\models_{M}$ inductively as follows. Let $\mathcal{M}=\langle\mathcal{F}, V\rangle$ be a model where $\mathcal{F} \in \mathcal{K}^{U}$ and $V$ is a valuation function, and let $w \in W$. For constants, proposition symbols and Boolean connectives $\models_{M}$ is defined as $\models$ in Kripke semantics. Let $\varphi$ be a modal formula. If $w \in U$ then

$$
\mathcal{M}, w \models_{M} \square \varphi \text { if and only if }\left|R[w] \cap\|\varphi\|_{M}^{\mathcal{M}}\right| \text { is odd, }
$$

and if $w \notin U$ then

$$
\mathcal{M}, w \models_{M} \square \varphi \text { if and only if }\left|R[w] \cap\|\varphi\|_{M}^{\mathcal{M}}\right| \text { is even. }
$$

Here $\|\varphi\|_{M}^{\mathcal{M}}$ denotes the truth set of $\varphi$ in the model $\mathcal{M}$ with respect to $\models_{M}$.

We denote by $\mathcal{L}$ the pair $\left(\mathcal{K}^{U}, \models_{M}\right)$. Let $\mathcal{K}_{O}^{U}$ be the class of all frames $\langle W, R, U\rangle$ in $\mathcal{K}^{U}$ such that every $w \in W$ has an odd number of $R$-successors. We denote by $\mathcal{L}_{S}$ the pair $\left(\mathcal{K}_{O}^{U}, \models_{M}\right)$.

The addition of a unary relation also affects the definition of corresponding classes. Instead of translating a binary relation to a set function and vice versa, we also have to take into account the unary relation defined on the universe. Let $\mathcal{K}^{\prime}$ be a subclass of $\mathcal{K}^{U}$ and let $\mathcal{C}$ be a class of Scott-Montague frames. We say that the pair $\left(\mathcal{K}^{\prime}, \models_{M}\right)$ corresponds to the class $\mathcal{C}$ if there are translations $(R, U) \mapsto F_{(R, U)}$ and $F \mapsto\left(R_{F}, U_{F}\right)$ such that the following conditions hold.

- If $\langle W, R, U\rangle \in \mathcal{K}^{\prime}$, then $\left\langle W, F_{(R, U)}\right\rangle \in \mathcal{C}$, and for all valuations $V$, the models $\langle W, R, U, V\rangle$ and $\left\langle W, F_{(R, U)}, V\right\rangle$ are pointwise equivalent with respect to $\models_{M}$.

- If $\langle W, F\rangle \in \mathcal{C}$, then $\left\langle W, R_{F}, U_{F}\right\rangle \in \mathcal{K}^{\prime}$, and for all valuations $V$, the models $\left\langle W, R_{F}, U_{F}, V\right\rangle$ and $\langle W, F, V\rangle$ are pointwise equivalent with respect to $\models_{M}$.

- $R_{F_{(R, U)}}=R, U_{F_{(R, U)}}=U$ and $F_{\left(R_{F}, U_{F}\right)}=F$.

Furthermore, we say that the pair $\left(\mathcal{K}^{\prime}, \models_{M}\right)$ corresponds to a clone $C$ if the pair $\left(\mathcal{K}^{\prime}, \models_{M}\right)$ corresponds to the class $\mathcal{C}_{C}$.

Proposition 10. The pair $\mathcal{L}$ corresponds to the clone $L$.

Proof. The clone $L$ is defined by the functional term

$$
\mathbf{1} \oplus \mathbf{f}(\mathbf{0}) \oplus \mathbf{f}(\mathbf{x}) \oplus \mathbf{f}(\mathbf{y}) \oplus \mathbf{f}(\mathbf{x} \oplus \mathbf{y}) .
$$


Thus the class $\mathcal{C}_{L}$ of Scott-Montague frames is definable by the axiom $\top \oplus \square \perp \oplus$ $\square p \oplus \square q \oplus \square(p \oplus q)$. This axiom is valid in a Scott-Montague frame $\mathcal{F}=\langle W, F\rangle$ if and only if the condition

(i) $F(\emptyset) \oplus F(X) \oplus F(Y)=F(X \oplus Y)$

holds for all $X, Y \subseteq W$.

The translations $(R, U) \mapsto F_{(R, U)}$ and $F \mapsto\left(R_{F}, U_{F}\right)$ are defined as follows. For all $X \subseteq W$,

$$
\begin{aligned}
F_{(R, U)}(X)= & \{w \in W \mid w \in U \text { and }|R[w] \cap X| \text { is odd }\} \cup \\
& \{w \in W \mid w \notin U \text { and }|R[w] \cap X| \text { is even }\},
\end{aligned}
$$

$R_{F}$ is defined as before Lemma 8 and $U_{F}=\{w \in W \mid w \notin F(\emptyset)\}$.

Let $\langle W, R, U\rangle \in \mathcal{K}^{U}$. As in the proof of Proposition 7, we can show that for all $X, Y \subseteq W$ and for all $w \in W$,

$$
w \in F_{(R, U)}(\emptyset) \oplus F_{(R, U)}(X) \oplus F_{(R, U)}(Y) \text { if and only if } w \in F_{(R, U)}(X \oplus Y) .
$$

Thus $F_{(R, U)}$ satisfies the condition (i) and $\left\langle W, F_{(R, U)}\right\rangle \in \mathcal{C}_{L}$.

Let $V$ be a valuation function and let $\mathcal{M}=\langle W, R, U, V\rangle$ and $\mathcal{M}^{\prime}=\left\langle W, F_{(R, U)}, V\right\rangle$. We show that for all modal formulas $\varphi$ and for all $w \in W$,

$$
\mathcal{M}, w \models_{M} \varphi \text { if and only if } \mathcal{M}^{\prime}, w \models \varphi .
$$

The proof is by induction on the construction of $\varphi$. We consider only the case where $\varphi$ is of the form $\square \psi$, the other ones are straightforward. So let $\varphi=\square \psi$ and suppose that the claim holds for $\psi$, whence $\|\psi\|_{M}^{\mathcal{M}}=\|\psi\|^{\mathcal{M}^{\prime}}$. Let $w \in U$. Then

$$
\begin{aligned}
\mathcal{M}, w \models_{M} \square \psi & \Leftrightarrow\left|R[w] \cap\|\psi\|_{M}^{\mathcal{M}}\right| \text { is odd } \\
& \Leftrightarrow\left|R[w] \cap\|\psi\|^{\mathcal{M}^{\prime}}\right| \text { is odd } \\
& \Leftrightarrow w \in F_{(R, U)}\left(\|\psi\|^{\mathcal{M}^{\prime}}\right) \\
& \Leftrightarrow \mathcal{M}^{\prime}, w \models \square \psi .
\end{aligned}
$$

Similarly, $\mathcal{M}, w \models_{M} \square \psi$ if and only if $\mathcal{M}^{\prime}, w \models \square \psi$, whenever $w \notin U$. Thus the models $\mathcal{M}$ and $\mathcal{M}^{\prime}$ are pointwise equivalent with respect to $\models_{M}$.

Let $\langle W, F\rangle \in \mathcal{C}_{L}$. Since $R_{F}$ is a binary relation on $W$ and $U_{F}$ is a subset of $W$, we have that $\left\langle W, R_{F}, U_{F}\right\rangle \in \mathcal{K}^{U}$.

Let $V$ be a valuation and let $\mathcal{M}=\langle W, F\rangle$ and $\mathcal{M}^{\prime}=\left\langle W, R_{F}, U_{F}\right\rangle$. As above, it can be shown that the models $\mathcal{M}^{\prime}$ and $\mathcal{M}$ are pointwise equivalent with respect to $\models_{M}$.

Let $\langle W, R, U\rangle \in \mathcal{K}^{U}$ and $\langle W, F\rangle \in \mathcal{C}_{L}$. We still have show that $R_{F_{(R, U)}}=R$, $U_{F_{(R, U)}}=U$ and $F_{\left(R_{F}, U_{F}\right)}=F$. Let $w, v \in W$. It is easy to see that

$$
w \in U \Leftrightarrow w \notin F_{(R, U)}(\emptyset) \Leftrightarrow w \in U_{F_{(R, U)}} .
$$

Also, it is not difficult to see that

$$
\begin{aligned}
v \in R[w] & \Leftrightarrow w \in \bar{U} \oplus F_{(R, U)}(\{v\}) \\
& \Leftrightarrow w \in F_{(R, U)}(\emptyset) \oplus F_{(R, U)}(\{v\}) \\
& \Leftrightarrow v \in R_{F_{(R, U)}}[w] .
\end{aligned}
$$

Thus $R_{F_{(R, U)}}=R$ and $U_{F_{(R, U)}}=U$. 
Let $X \subseteq W$ and $w \in W$. Suppose that $w \in F_{\left(R_{F}, U_{F}\right)}(X)$. Let $w \in F_{\left(R_{F}, U_{F}\right)}(\emptyset)$. Then $w \notin U_{F}$ and furthermore $w \in F(\emptyset)$. Since $w \notin U_{F}$ and $w \in F_{\left(R_{F}, U_{F}\right)}(X)$, we can conclude that $\left|R_{F}[w] \cap X\right|$ is even. Since $w \in F(\emptyset)$, it follows from Lemma 8 that $w \in F(X)$. Similarly we can prove that $w \in F(X)$ if $w \notin F_{\left(R_{F}, U_{F}\right)}(\emptyset)$.

Let $w \in F(X)$. Assume first that $w \in F(\emptyset)$. Then $w \notin U_{F}$. Since $w \in F(\emptyset)$ and $w \in F(X)$, it follows from Lemma 8 that $\left|R_{F}[w] \cap X\right|$ is even, and hence we get that $w \in F_{\left(R_{F}, U_{F}\right)}(X)$. A similar proof shows that $w \in F_{\left(R_{F}, U_{F}\right)}(X)$ when $w \notin F(\emptyset)$. Thus $F_{\left(R_{F}, U_{F}\right)}=F$.

With similar methods, using the same translations $(R, U) \mapsto F_{(R, U)}$ and $F \mapsto$ $\left(R_{F}, U_{F}\right)$ as in the proof of Proposition 10, we can also prove the following proposition which completes the cases of linear clones.

Proposition 11. The pair $\mathcal{L}_{S}$ corresponds to the clone $L S$.

\section{Conclusion}

We have established a complete correspondence between definability of Boolean functions by functional terms and definability of finite Scott-Montague frames by uniform degree-1 formulas. We introduced a bijective translation between functional terms and uniform degree-1 formulas and, based on this, we showed that a class of Boolean functions is defined by functional terms if and only if the corresponding class of Scott-Montague frames is defined by the translations of these functional terms, and vice versa. Furthermore, we characterized classes of ScottMontague frames corresponding to the clone $\Lambda_{1}$ and to some particular subclones of $\Lambda_{1}$ by classes of Kripke frames. By modifying Kripke semantics, we were also able to characterize the classes of Scott-Montague frames corresponding to the linear clones $L, L_{0}, L_{1}, L_{c}$ and $L S$.

At this point the obvious question is: For which of the remaining clones the corresponding class of Scott-Montague frames can be characterized by further modifications of Kripke semantics? In the bottom of Post Lattice, we still lack characterizations for the classes of frames corresponding to the clones $V, V_{1}, \Lambda, \Lambda_{0}, \Omega(1), I$ and $I^{*}$. Our intention is to study these cases in a subsequent paper. It seems that some of them can be obtained from the results in this paper by using some minor technical tricks.

However, some interesting clones, such as $S, M$ and $S M$, seem to be more difficult to handle. In the case of the bottom clones and linear clones, the associativity of the generating functions of the clones seems to be crucial. There is a qualitative difference when associativity does not hold. For example, the clone $S M$ is generated by the majority of three variables, which is not an associative function. Nevertheless, we are going to study in a follow-up paper whether the classes of Scott-Montague frames corresponding to the clones in the top of Post Lattice can also be characterized by modifying Kripke semantics in a different way. 


\section{REFERENCES}

[1] P. Blackburn, M. de Rijke, Y. Venema. Modal Logic, Cambridge University Press, Cambridge, 2001.

[2] F. M. Brown. Boolean Reasoning: The Logic of Boolean Equations, 2nd ed., Dover, Mineola, New York, 2003.

[3] B. F. Chellas. Modal Logic, an Introduction, Cambridge University Press, 1980.

[4] M. Couceiro, S. Foldes. "Function Class Composition, Relational Constraints and Stability under Compositions with Clones", Rutcor Research Report 22-2004, Rutgers University, http://rutcor.rutgers.edu/ rrr/.

[5] M. Couceiro, S. Foldes. "Constraints, Functional Equations, Definability of Function Classes, and Functions of Boolean Variables", Rutcor Research Report 36-2004, Rutgers University, http://rutcor.rutgers.edu/ rrr/.

[6] M. Couceiro, S. Foldes, E. Lehtonen. "Composition of Post Classes and Normal Forms of Boolean Functions", Rutcor Research Report 05-2005, Rutgers University, http://rutcor.rutgers. edu/ rrr/.

[7] O. Ekin, S. Foldes, P. L. Hammer, L. Hellerstein. "Equational Characterizations of Boolean Functions Classes", Discrete Mathematics, 211 (2000) 27-51.

[8] S. Foldes, G. Pogosyan. "Post Classes Characterized by Functional Terms", Discrete Applied Mathematics 142 (2004) 35-51

[9] B. Hansson, P. Gärdenfors "A Guide to Intensional Semantics", Modality, Morality and Other Problems of Sense and Nonsense: Essays Dedicated to Sören Halldén, ss. 151-67, Gleerup, Lund, 1973.

[10] S. W. Jablonski, G. P. Gawrilow, W. B. Kudrjawzew. Boolesche Funktionen und Postsche Klassen, Vieweg, Braunschweig, 1970.

[11] N. Pippenger. Theories of Computability, Cambridge University Press, Cambridge, 1997.

[12] N. Pippenger. "Galois Theory for Minors of Finite Functions", Discrete Mathematics, 254 (2002) 405-419.

[13] G. Pogosyan. "Classes of Boolean Functions Defined by Functional Terms", Multiple Valued Logic, 7 (2002) 417-448

[14] E. L. Post. "The Two-Valued Iterative Systems of Mathematical Logic", Annals of Mathematical Studies 5 (1941) 1-122.

[15] M. Reschke, K. Denecke. "Ein neuer Beweis fr die Ergebnisse von E. L. Post ber abgeschlossene Klassen Boolescher Funktionen", Elektronische Informationsverarbeitung und Kybernetik 25(7) (1989) 361-380.

[16] K. Segerberg. "An Essay in Classical Modal Logic", Filosofiska Studier utgivna av Filosofiska Freningen och Filosofiska Institutionen vid Uppsala Universitet, nr 13, Uppsala, 1971.

[17] M. H. Stone. "Subsumption of the Theory of Boolean Algebras under the Theory of Rings", Proceedings of the National Academy of Sciences USA, 31 (1935) 103-105.

[18] A. B. Ugolnikov. "Closed Post Classes" (in Russian), Izvestiya VUZ. Matematika 32 (1988) 79-88.

[19] A. Virtanen. "Essee Deskriptiivisestä Modaalilogiikasta", manuscript, Tampere, 1994

[20] I. E. Zverovich. "Characterizations of Closed Classes of Boolean Functions in Terms of Forbidden Subfunctions and Post Classes", Rutcor Research Report 17-2000, Rutgers University, http://rutcor.rutgers.edu/ rrr/. To appear in Discrete Applied Mathematics.

Department of Mathematics, Statistics and Philosophy, University of Tampere, KaleVANTIE 4, 33014 TAMPERE, FinLand 